\title{
ROOT DISTRIBUTION OF 430 PLANTS IN TEMPERATE GRASSLAND OF NORTHERN CHINA
}

\author{
WANG, Z. Y. ${ }^{1, \uparrow}-$ HOU, J. ${ }^{2, \uparrow}-$ QU, Z. Q. ${ }^{1}-$ GUO, J. Y. ${ }^{3, *}-$ LI , J. R. ${ }^{3}$ \\ ${ }^{1}$ College of Grassland, Resource and Environment, Inner Mongolia Agriculture University \\ 306 Zhaowuda Road, Saihan district, Hohhot, China \\ (tel /fax: +86-471-4316259) \\ ${ }^{2}$ College of Vocational and Technical, Inner Mongolia Agriculture University \\ 306 Zhaowuda Road, Saihan district, Hohhot, China \\ (tel /fax: +86-471-4316259) \\ ${ }^{3}$ Institute of Hydraulic Research in Pastoral Areas, Chinese Ministry of Water Resources \\ 128 Daxuedong Road, Saihan District, Hohhot, China \\ (tel /fax: +86-471-3970128) \\ ${ }^{\dagger}$ These authors contributed equally to this article. \\ *Corresponding author \\ e-mail: guojianying1980@163.com \\ (Received 13 $3^{\text {th }}$ Jan 2017; accepted $4^{\text {th }}$ Apr 2017)
}

\begin{abstract}
Plants in arid environments tend to have large, deep root systems, so plants living in different environments may have different root morphology. How many types of plant roots exist in the grassland? To answer this question, root morphology of each plant species should be observed, and then summarized. However, very limited data considering root distribution of each grassland species is available due to methods limitation. In this study, 430 plant species (including 542 observations) were selected. The study found that the majority of plant roots in grassland of northern China can be divided into six types: rhizome-rooted plant, fasciculate rooted plants, creeping rooted plants, fibrous rooted plants, tap-rooted plants, the bulb-corm-tuber rooted type. Based on the root types, the average root depth is $63.84 \pm 5.96 \mathrm{~cm}$ for tap rooted, $49.89 \pm 7.06 \mathrm{~cm}$ for creeping rooted, $32.03 \pm 4.29 \mathrm{~cm}$ for fasciculate rooted, $23.72 \pm 2.77 \mathrm{~cm}$ for rhizome rooted, $20.38 \pm 3.83 \mathrm{~cm}$ for fibrous rooted, $15.79 \pm 2.02 \mathrm{~cm}$ for bulbcorm-tuber rooted plants. The unilateral root spread is $44.58 \pm 9.26 \mathrm{~cm}$ for tap rooted, $43.12 \pm 7.64 \mathrm{~cm}$ for creeping rooted, $23.82 \pm 2.79 \mathrm{~cm}$ for rhizome rooted, $21.00 \pm 2.93 \mathrm{~cm}$ for fasciculate rooted, $13.86 \pm 2.64$ $\mathrm{cm}$ for fibrous rooted, $12.64 \pm 2.44 \mathrm{~cm}$ for bulb-corm-tuber rooted plants. It was found that plants with deeper tap root and longer lateral roots are mainly distributed in sandy and desert habitats. Plants with shallow tap roots and short lateral roots are mainly distributed in humid meadows, marsh soil and meadow soil. In humid regions, some plants were found to have a very large root system and at the same time large aboveground biomass.
\end{abstract}

Keywords: root depth, unilateral roots, steppe, root type, height

\section{Introduction}

When compared with the research concearning aboveground plant parts, the research in plant roots and belowground organs are relatively lacking. Mainly, because it is relatively difficult to sample and observe the root buried in soil. We are aware, that complete root system can only be obtained through the excavation method, yet this method is laborious and time-consuming. Additionally, there are many kinds of plants distributed widely. Therefore, the current data on root morphology is limited to only some 
species. Efforts to understand the root morphological characteristics of all the plants, should be made through worldwide and long-term cooperation of scientists.

On this account, professor Chen Shihuang, had been aquiring 430 kinds of plant roots and had been gathering observational data for the past 50 years. Because of the identical way of collecting, the results can be well compared. The collected data was analyzed and is summerized in this article.

\section{Review of Literature}

Drought tolerance and water use efficiency of plants are important because aridity in many areas of the world severely limits plant growth. Aridity is expected to become more severe, especially in arid and semi-arid areas of the world, such as in central and northern China (Zhang et al., 2016; Fu and Guo, 2008). The depth, lateral spread and degree of overlap of plant root systems are important for plant's adsorption of water and nutrients (Casper and Jackson, 1997). Plants in arid areas have particurally large belowground biomass. According to Ma and Fang (2006), belowground biomass of plants in Inner Mongolian grasslands is six to ten times greater than aboveground biomass. Studying the distribution of plant roots is useful to predict different responses of plants under future drought conditions, and in order to develop strategies to protect local ecological environment and maintain ecological functions in the grassland.

Study of the vertical and horizontal distribution of roots is of particular ecological significance. Most of plant root biomass occurs in the $0-50 \mathrm{~cm}$ soil layer, and only a small portion reaches deep soil (for a review see Canadell et al. (1996)). Therefore, it has commonly been assumed that study of plant roots in the $50 \mathrm{~cm}$ soil layer can allow to fully understand the function and structure of the root systems on the ecosystem level. However, more and more research has shown the significant role of deep roots, especially for water, carbon and nutrient cycling (Douglas, 2011; Philippot et al., 2009; Yu et al., 2015).

Elongation of lateral roots of adjacent plants can affect the amount of available resources in an ecosystem and thus determine the competitive advantage between plants (Jose et al., 2003; Ruta et al., 2010; Wang et al., 2002). Data on root depth and lateral root spread can help predicting functional differences between plants with different growth under future climate change scenarios. However, the absolute root depth or horizontal extension of roots may even be greater in humid environments, because plants shoots can be relatively large in these regions (Schenk and Jackson, 2002). Study of the vertical and horizontal distribution characteristics of plant roots can provide important reference values for plant root sampling techniques, and can be useful in building models related to roots; and in calculating the carbon retention rates in global grasslands.

Roots are buried in the soil, but soil is an opaque and heterogeneous composite medium containing a lot of gravel. This increases the difficulty of root sampling and research. Therefore, many scholars have tried to employ new methods to study root systems, including the excavation method, the profile method, the root window method, ground penetrating radar and isotope labeling methods.

Compared to other methods, the excavation method is the only method in which relatively intact roots can be obtained. The excavation method is suitable for sampling shallow roots of herbs, but is very laborious and time-consuming when applied to the sampling of shrub and tree roots. Despite the difficulty, inefficiency and labor requirements, we need to use the excavation method to see the full morphology of a root 
system. The plant roots research team led by Chen Shihuang had accumulated abundant primary research data on plants' roots in grasslands over the past 50 years, including data on the basic morphology, depth and horizontal extension of roots in relation to the plants' habitat.

We analyzed the data in order to test some previously formed hypotheses: 1) plants have deeper or wider roots in water deficient environments, and also have deeper or wider roots under water-abundant conditions; 2) root morphology and root types are related to steppe habitats. The main root types in the steppe of northern China include tap roots, creeping roots, rhizome roots and fasciculate roots. This analysis aims to provide the basis for further study of belowground part of grassland ecosystem.

\section{Materials and Methods}

Plant roots were mostly sampled from Inner Mongolia Autonomous Region and northeast China, with some samples from Xinjiang Autonomous Region and Gansu Province (Figure 1, more details about the sites for each species can be acquired from supplements or from authors). Sampling was done at the beginning of August and at the end of July (which is the period of peak biomass). Typical plants were selected based on the growth of aboveground biomass, root samples were obtained using the excavation method. During the excavation process, it is necessary to obtain a root system, that is as complete as possible. The depth and horizontal distribution of roots and plant height were then measured in the field with a ruler, and the morphology of the roots was drawn on paper. A few observations of plant roots were collected from a special local sites, such as landslide area or a river bank. We analyzed 541 observations of plant roots, which covered 430 species of plants in different habitats. In some cases plants of the same species had different root morphology in different habitats.

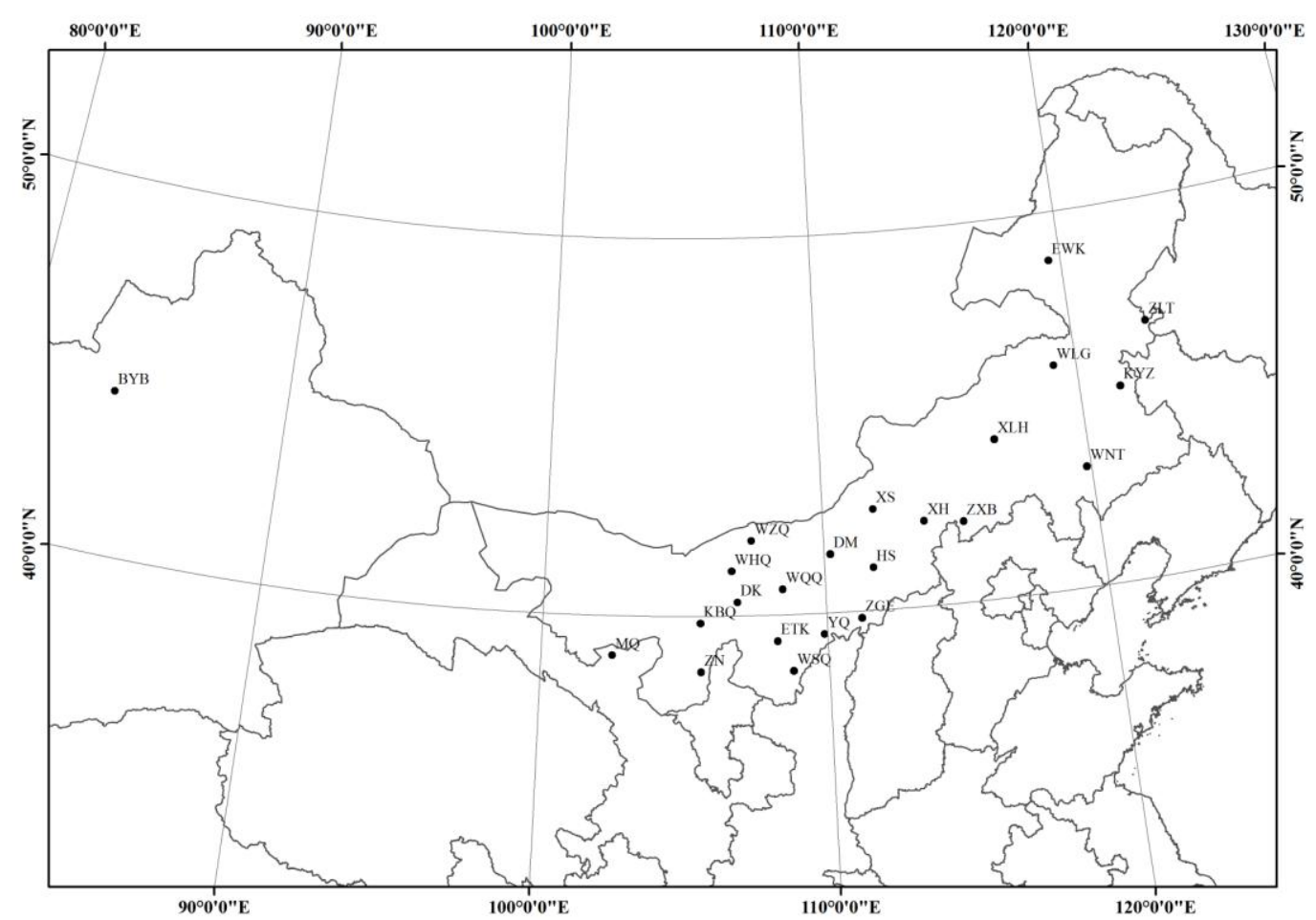

Figure 1. The sampling sites of the plant in this study 
The plants were divided into six root types based on the root morphology. Rhizomerooted plants have a subterranean stem (Figure 2a). Creeping rooted plants are characterized by a horizontal root with branches coming from the horizontal root (Figure $2 c$ ), while fasciculate rooted plants have many branches of the same size coming from both the root and shoots (Figure $2 b$ ). Fibrous rooted plants have a single long stem with many of thin roots of the same thickness (Figure $2 d$ ). Tap-rooted plants exhibit a significant difference between the diameter of the main root and lateral roots (Figure 2e). The bulb-corm-tuber rooted type refers to plants that have roots with abnormal organs such as tubers, corms or bulbs (Figure 2f). More details about the root classification can be found in Liu and Chen (2001) and Armstrong (2001).

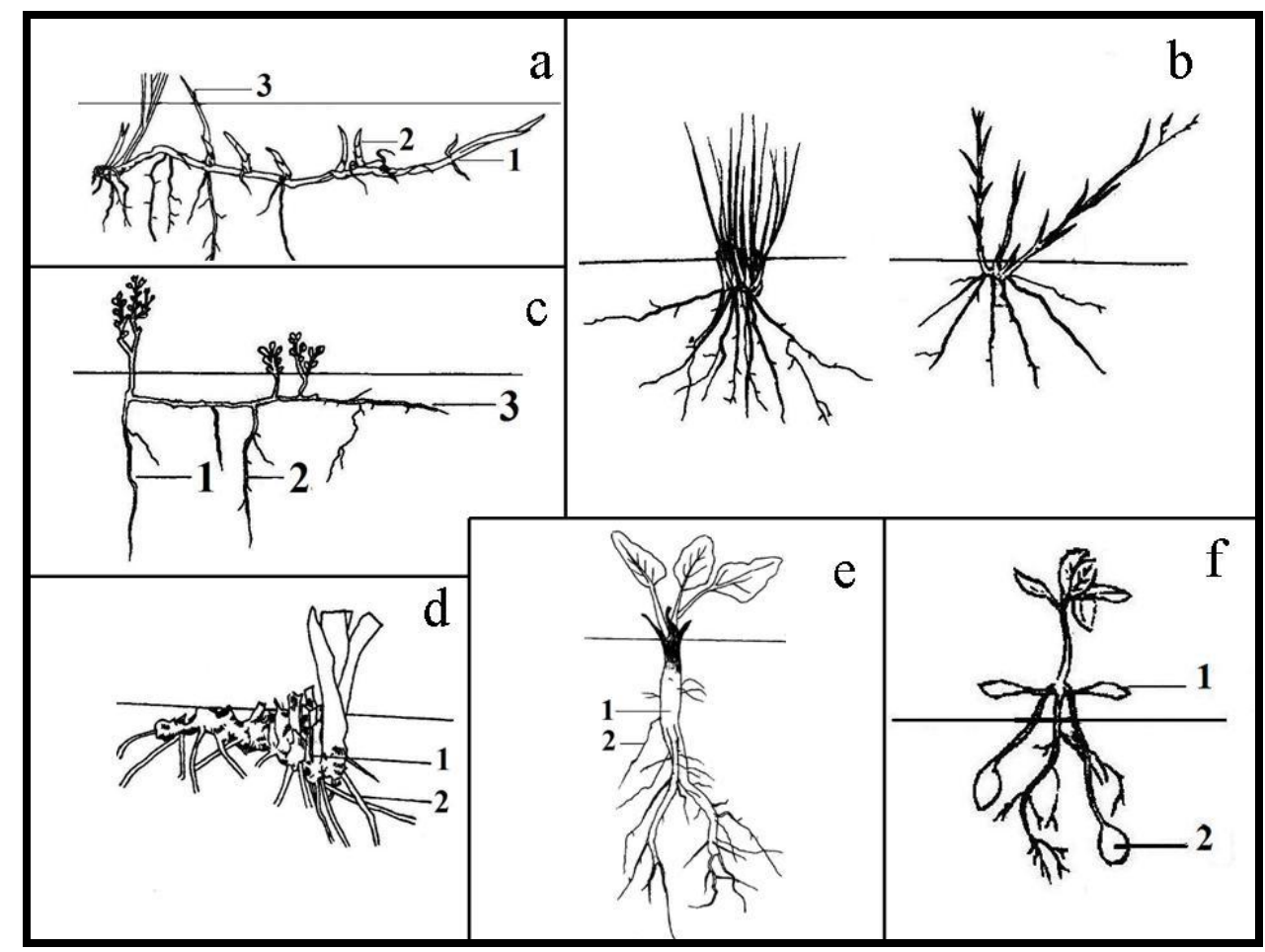

Figure 2. The different root types of plants found in grassland of northern China. note: a. Rhizome-rooted plant (1, subterranean stem; 2,bud; 3, shoot); b. Fasciculate rooted plants; $c$.

Creeping rooted plants (1, main root;2, vertical root; 3 , horizontal root); $d$. Fibrous rooted plants; e.Tap-rooted plants (1, main root;2,lateral roots); $f$. The bulb-corm-tuber rooted type (1, shoot; 2, abnormal organs)

\section{Results}

\section{Root depth of grassland plants}

The average root depth of different life forms is shown in Figure 3. The average root depth of shrubs is $140.03 \pm 14.63 \mathrm{~cm}(\mathrm{n}=38)$, with Caragana intermedia Kuang. (620 $\mathrm{cm})$ being the longest. The average root depth of semi-shrubs is $100.14 \pm 25.86 \mathrm{~cm}(\mathrm{n}=$ 50), the longest of which is Alhagi sparsifolia Shap. (12 m), which grows in the desert. The average root depth of perennial herbs is $38.01 \pm 2.41 \mathrm{~cm}(\mathrm{n}=367)$, the longest of which is Medicago sativa L. (467 cm). The average root depth of annual herbs is 18.24 $\pm 1.98 \mathrm{~cm}(\mathrm{n}=86)$, the longest of which is Dracocephalum moldavica L. $(96.67 \mathrm{~cm})$, which grows in fixed sand. 


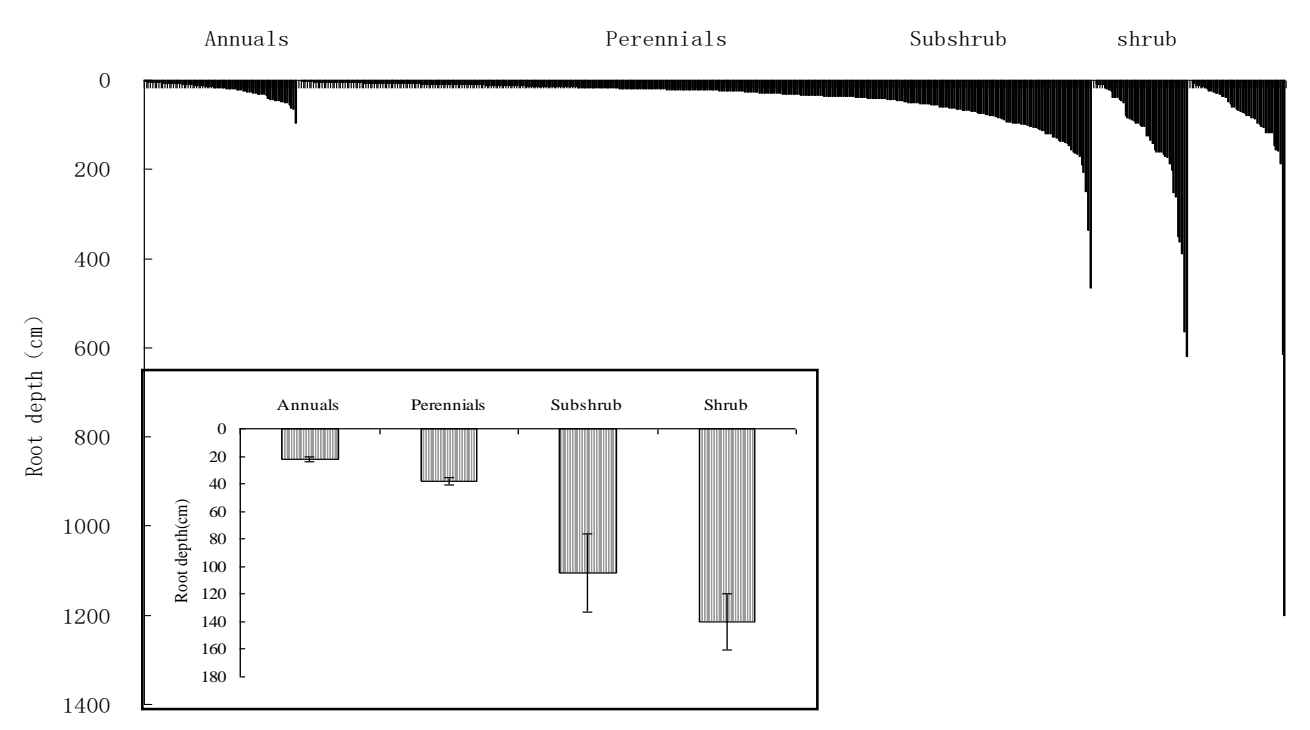

Figure 3. Root depth of plants with different life forms

In this study, a total of 541 observations of root depth were used. Most plant roots are distributed in the 0 to $50 \mathrm{~cm}$ soil layer, accounting for $72.09 \%$ of the total observations (Table 1). Sixty six observations of root data were distributed deeper than $100 \mathrm{~cm}$. These observations came from 60 kinds of plants, including perennial herbs ( 27 species), shrubs (18 species), semi-shrubs (10 species), small shrubs (3), small trees (1) and biennial plants (1). Among these observations, 42 observations were sampled from sandy and arid soils, and 7 from salinized soil and wet meadow environments.

Table 1. Root distribution interval of 541 data observations used in this study

\begin{tabular}{|c|c|c|c|c|c|c|c|c|c|}
\hline \multirow{4}{*}{$\begin{array}{c}\text { Depth } \\
\text { (proportion) } \\
\text { Lateral root } \\
\text { spread } \\
\text { (proportion) }\end{array}$} & \multicolumn{9}{|c|}{ Data observations (proportion \%) } \\
\hline & $0 \leq \mathrm{L}^{*}<0.5$ & $0.5 \leq \mathrm{L}<1$ & $1 \leq \mathrm{L}<1.5$ & $1.5 \leq \mathrm{L}<2$ & $2 \leq \mathrm{L}<3$ & $3 \leq \mathrm{L}<4$ & $4 \leq \mathrm{L}<5$ & $\mathrm{~L}>5$ & Sum \\
\hline & $\begin{array}{c}390 \\
(72.09 \%)\end{array}$ & $\begin{array}{c}85 \\
(15.71 \%)\end{array}$ & $\begin{array}{c}35 \\
(6.47 \%)\end{array}$ & $\begin{array}{c}17 \\
(3.14 \%)\end{array}$ & $\begin{array}{c}5 \\
(0.92 \%)\end{array}$ & $\begin{array}{c}4 \\
(0.74)\end{array}$ & $\begin{array}{c}1 \\
(0.18 \%)\end{array}$ & $\begin{array}{c}4 \\
(0.74)\end{array}$ & 541 \\
\hline & $\begin{array}{c}472 \\
(87.41 \%)\end{array}$ & $\begin{array}{c}40 \\
(7.41 \%)\end{array}$ & $\begin{array}{c}9 \\
(1.67 \%)\end{array}$ & $\begin{array}{c}7 \\
(1.30 \%)\end{array}$ & $\begin{array}{c}5 \\
(0.93 \%)\end{array}$ & $\begin{array}{c}2 \\
(0.31)\end{array}$ & $\begin{array}{c}1 \\
(0.19 \%)\end{array}$ & $\begin{array}{c}4 \\
(0.74)\end{array}$ & 540 \\
\hline
\end{tabular}

* "L" indicates length in meters.

The roots in 85 observations were distributed between $50-99 \mathrm{~cm}$ deep. These observations include data from 80 species: annual and biennial plants (7 species), perennials plants (53 species), and shrubs, semi-shrubs and dunga-runga (20 species). Of these 85 observations, 31 observations were from plants sampled in sandy soils.

There were 114 observations from a soil depth of $25-50 \mathrm{~cm}$, including 105 species of annual and biennial plants (20 species), perennials plants (73 species), shrubs, semishrubs, undershrubs and sub-shrubs (12 species). Out of these, 46 observations came from sandy soils.

There were 276 observations from a root depth of $0-25 \mathrm{~cm}$, including 243 species. Out of these observations, 136 were distributed in humid, aquatic or river bank environments. 
The root depth of each root type is shown in Figure 3. The depth of tap rooted plants is $63.44 \pm 5.97 \mathrm{~cm}(\mathrm{n}=311)$ on average, of which the root of Alhagi sparsifolia Shap. is the deepest. The average depth of creeping rooted plants is $52.56 \pm 7.12 \mathrm{~cm}(\mathrm{n}=55)$, of which the root of Thermopsis lanceolata $\mathrm{R}$. Br., growing in semi-fixed sand, is the deepest $(248.89 \mathrm{~cm})$. The average depth of fasciculate rooted plants is $32.03 \pm 3.33 \mathrm{~cm}$ $(\mathrm{n}=51)$, of which the root of Stipa baicalensis Roshev., which grows in dark chestnut soils, is the deepest $(113.44 \mathrm{~cm})$. The depth of rhizome rooted plants is $23.72 \pm 2.77 \mathrm{~cm}$ ( $n=69)$ on average, of which the root of Psammochloa villosa (Trin.), which is found growing in sand dunes, is the deepest $(120 \mathrm{~cm})$. The average depth of fibrous rooted plants is $20.38 \pm 3.83 \mathrm{~cm}(\mathrm{n}=30)$, of which the root of Asparagus gobicus Ivan. ex Grub. is the deepest $(116.84 \mathrm{~cm})$. The depth of tuber-bulb rooted plants is $15.79 \pm 2.02$ $\mathrm{cm}(\mathrm{n}=24)$ on average, among which the root of Allium ramosum $\mathrm{L}$., which grows in the litho soils found in hilly areas of meadow steppe, is the deepest $(37.14 \mathrm{~cm})$.

\section{Unilateral root spread}

Based on the life form (Figure 4), the average unilateral root spread of annual plants is $11.88 \pm 1.07 \mathrm{~cm}$, of which the root of Melilotus officinalis (Linn.) Pall. is the longest $(48.89 \mathrm{~cm})$. The lateral root spread of perennials is $19.94 \pm 1.20 \mathrm{~cm}$, of which Glycyrrhiza uralensis Fisch. $(223.5 \mathrm{~cm})$ and Ephedra sinica Stapf. $(225 \mathrm{~cm})$ have the longest horizontal extension. The lateral root spread of semi-shrubs is $71.20 \pm 15.82 \mathrm{~cm}$, of which the root of Alhagi sparsifolia Shap. growing in desert areas, extends the longest $(6 \mathrm{~m})$. The lateral root spread of shrubs is $186.49 \pm 60.29 \mathrm{~cm}$, of which the root of Salix gordejevii Y. L. Chang et Skv. extends the longest (20 m).

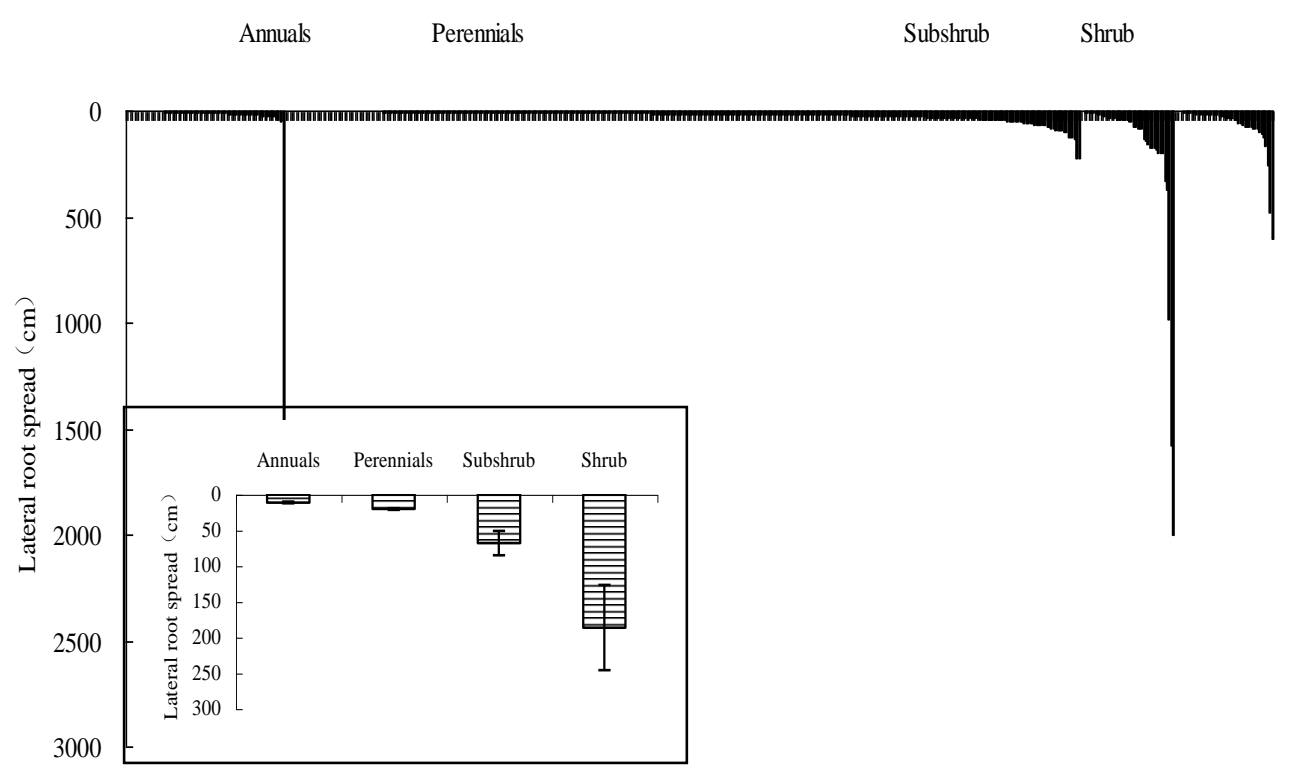

Figure 4. Lateral root spread of plants with different life form

There were 540 observation on lateral root spread. 472 observations were distributed between 0 to $50 \mathrm{~cm}$, representing $87.41 \%$ of the total observations (Table 1). 28 observations of lateral root spread reached $100 \mathrm{~cm}$, including 23 species of plants (4 
observations for perennials, 10 observations for shrubs, 5 observations for subshrubs, 3 observations for undershrubs, 1 observation for dunga-runga), of which 25 observations were located in sandy and desert areas.

The lateral root spread of 40 observations was between 50 and $100 \mathrm{~cm}$, including 40 species (22 perennial herbs, 5 shrubs, 11 semi-shrubs, 1 undershrub and 1 biennial plant). Out of the 40 observations, sandy, desert and brown soils contributed 16 observations, calcic soils, marsh and wetland soils, meadow soils and aquatic environments contributed 10 observations, light chestnut soils 9 observations, dark chestnut soils 4 observations, and a rocky hillside 1 observation.

The lateral root spread of 82 observations was between 25 and $50 \mathrm{~cm}$, covering 79 species. Of these observations, 35 were distributed in sandy soils and desert, and 29 in wet or salty soils, meadow or dark chestnut soils, 6 in light chestnut soils, 5 in chestnut soils, 4 in light brown calcic soil, 1 in alpine meadow of Tibet. Categorized by plant type, 9 observations were from annuals, 52 from perennial grasses, and 18 from shrubs, semi-shrubs and subshrubs.

The lateral root spread of 390 observations was between 0 and $25 \mathrm{~cm}$, covering 330 species, including 64 annual plants, 243 perennial herbs and 23 shrubs, semi-shrubs and undershrubs. Of the 390 observations, 186 observations were distributed in meadow or marsh soils and dark chestnut soils in meadow steppe and mountain areas, saline soil in river wetlands; 107 observations were from sandy soils, fixed dunes and desert areas; 49 observations from chestnut soils, light chestnut soils and dark chestnut soils; 18 observations from brown soils and 17 observations from stony soils and regosols.

The lateral root spread of roots categorized by root type is shown in Figure 5. The average lateral spread for tuber rooted plants is $23.82 \pm 2.78 \mathrm{~cm}$. The lateral root spread of Alisma orientale (G.Sam.) Juz. is the longest $(57.10 \mathrm{~cm}$ ). The average spread for fasciculate rooted plants is $20.99 \pm 2.93 \mathrm{~cm}$, of which Achnatherum splendens (Trin.) Nevski is the longest $(95 \mathrm{~cm})$. The average for rhizome rooted plants is $23.82 \pm 2.78 \mathrm{~cm}$, of which the lateral root spread of Acorus calamus L. is the longest $(121.70 \mathrm{~cm})$. The average lateral spread for tap rooted plants is $44.57 \pm 9.26 \mathrm{~cm}$, of which mature Salix gordejevii Y. L. Chang et Skv. is the longest $(2 \mathrm{~m})$. For creeping rooted plants, the average spread is $43.12 \pm 7.64 \mathrm{~cm}$, of which the lateral root spread of Hedysarum fruticosum Pall. Lignosum (Trautv.) Kitag. is the longest $(257.14 \mathrm{~cm})$.

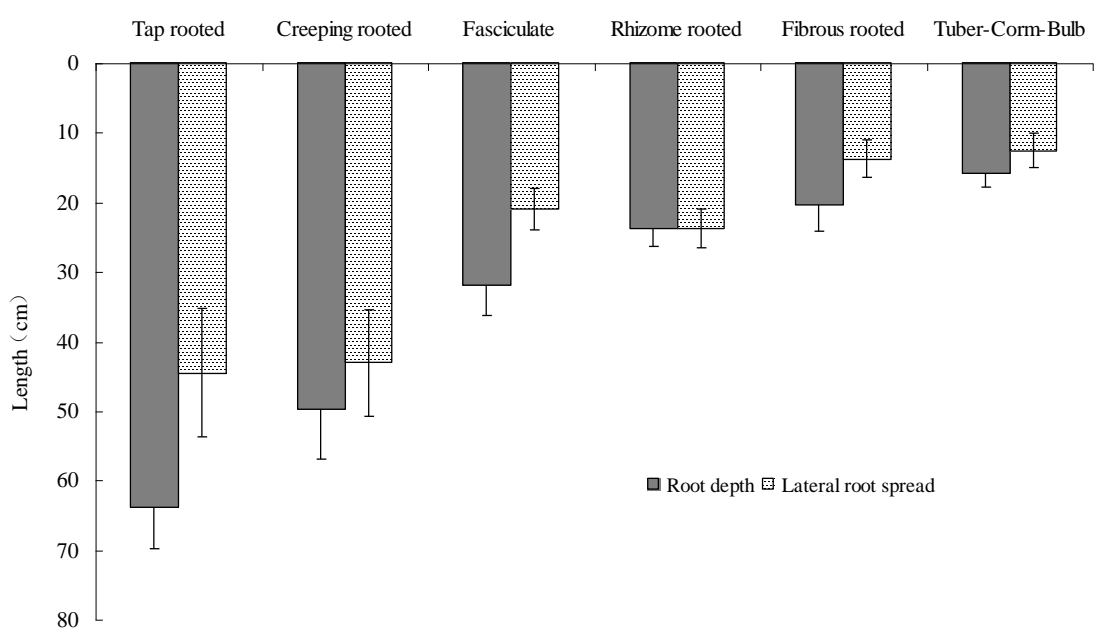

Figure 5. Depth and lateral root spread of plants with different root types 


\section{Discussion}

Using excavation method to remove the surrounding soil, the complete root morphology of plants was exposed. Since this method requires a large amount of physical labour and time (Smit et al., 2000), adequate data for root studies can only be collected through patient and persistent long-term data accumulation. Although necessary precautions were taken when exposing the roots during sampling process, damage to the roots was still difficult to avoid. Therefore, it is likely that the actual root depth and lateral root spread of some plants is greater than reported.

The root depth and lateral root spead of plants with different life forms follow the order: annuals < perennial herb < subshrub < various shrubs. This is in agreement with reports Schenk and Jackson (2002) and Canadell et al. (1996).

In this study, we classified the plant roots into 6 types (Liu and Chen, 2001). Relatively speaking, tap rooted plants can reach very deep into the soil. Creeping rooted plants have a strong capability to spread roots horizontally. Creeping rooted plants are mainly distributed in the desert steppe and the eastern part of the temperate steppedesert areas. Tap rooted plants are mainly distributed in forbrich steppe and the western part of the desert steppe zone. The root depth of fasciculate rooted plants is greater than that of rhizome rooted plants, and smaller than tap rooted or creeping rooted plants. Fasciculate rooted plants are mainly distrubted in typical steppe, while rhizome rooted types are mainly distrbuted in meadow steppe and typical steppe (Liu and Chen, 2001). Fibrous rooted and tuber-bulb rooted plants with subsidiary abnormal organs can store a lot of photosynthetic products, which can limit root growth vertically or horizontally.

Plant root depth and lateral root spread are related to habitat. In general, plants found in sandy and water-deficient environments have deeper roots. The deeper roots can absorb water from deep soils and increase plant's adaptation to drought (Yu et al., 2015). Sandy soils are aerated and loose, so roots can spread more easily. Though patterns of lateral root growth are species specific and habitat dependent, roots may be more likely to spread wider in sandy, nutrient-poor, and arid soils (Gour, 2006; Achten et al., 2008; Göttlicher et al., 2008).

Data on lateral root spread may be useful for estimation of belowground competition and the area over which plants interact with neighbours (Zamora et al., 2007; Christine et al., 2002). In this study, $87.41 \%$ of the total number of observations were distributed from 0 to $50 \mathrm{~cm}$. Shrubs and trees have a stronger ability to spread lateral root than herbs (Table 1), and most shrubs and trees sampled are located in arid areas.

However, we also found that some plants in the meadow steppe or under other humid conditions have large root systems. Some of these plants have a root deeper than $1 \mathrm{~m}$, such as the perennial plants Stipa grandis P. Smirn., Saussurea amara (L.) DC., Stipa baicalensis Roshev., Oxytropis myriophylla (Pall.) DC. and Hedysarum gmelinii Ledeb., Erodium stephanianum Willd., and Medicago sativa L. and shrubs such as Kalidium foliatum (Pall.) Moq., Kochia prostrata (L.) Schrad., Caragana microphylla Lam. and Caragana microphylla Lam. Var. cinerea Kom.. Some other plants, such as Glycyrrhiza uralensis Fisch., Ceratoides arborescens (Losinsk.) Tsien et C. G., Acorus calamus L., have lateral root spead of more than $100 \mathrm{~cm}$.

The plant root to shoot ratio $(\mathrm{R} / \mathrm{S})$ is related to the plant's habitat. Typically, plants have a higher R/S in water deficient soils (Woo et al., 2007; Neumann and Cardon, 2012). In this study, we found that plants living in sandy soils and water deficient environments have large root systems and low plant height, although some plants have large aboveground and belowground parts (Figure 6), such as Hedysarum fruticosum 
Pall. var. mongolicum Turcz. ex B. Fedtsch., Calligonum mongolicum Turcz., Myricaria platyphylla Maxim., Astragalus melilotoides Pall., Medicago sativa L., Salix gordejevii Y. L. Chang et Skv., Caragana korshinskii Kom., Alhagi sparsifolia Shap., and Haloxylon ammodendron (C. A. Mey.) Bunge. Most of these plants are trees and shrubs. Generally, R/S ratio and absolute maximum root depth of plants growing in arid regions are bigger than those of plants living in humid areas, although some studies have found the opposite (Schenk and Jackson, 2002; Mokany et al., 2006; Waring et al., 2017).

Regarding the plant root distribution pattern (Figure 6), one group of plants has very deep roots, while another group of plants has very long lateral roots, but no plants had both deep and long lateral roots. This may reflect two different survival strategies to obtain water, and thus seems reasonable from a resource allocation perspective.
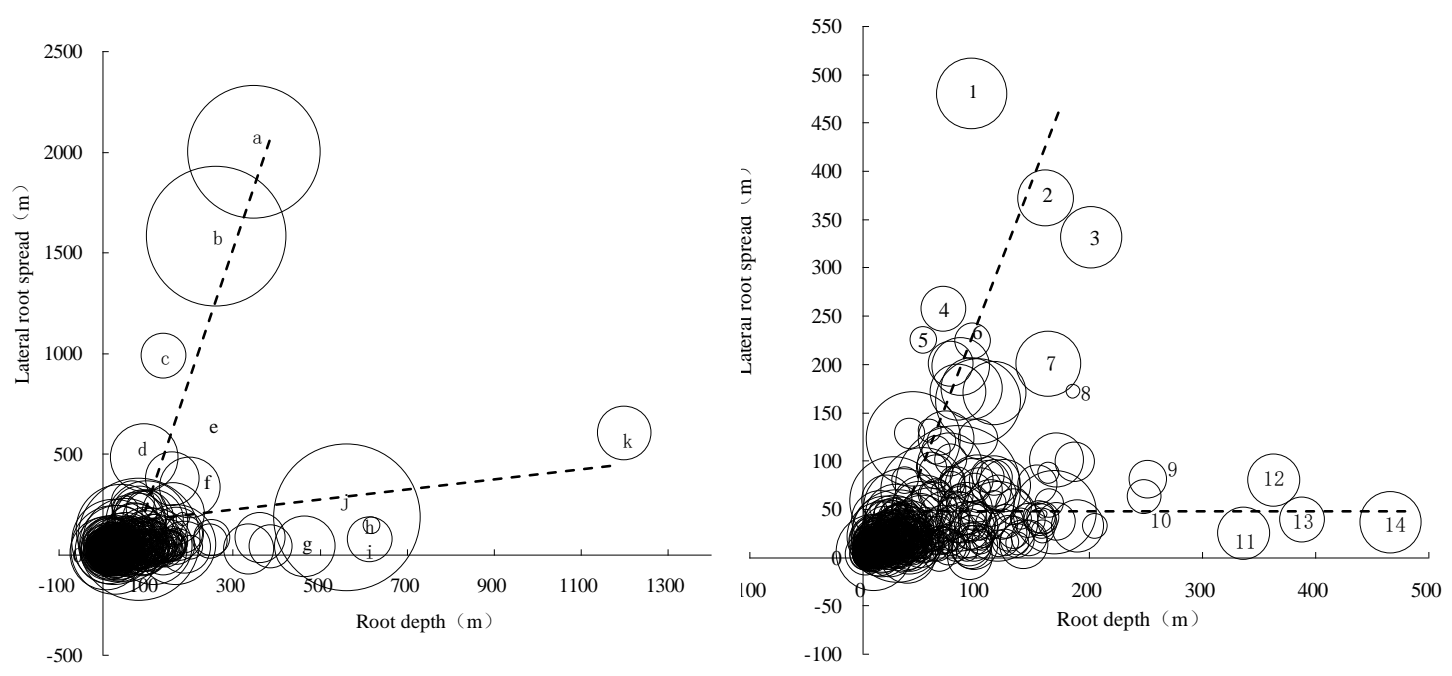

Figure 6. Height, depth and lateral root spread of plants

Right part: 1. Hedysarum fruticosum Pall. var. mongolicum Turcz. ex B. Fedtsch. 2. Calligonum mongolicum Turcz. 3. Myricaria platyphylla Maxim. 4. Hedysarum fruticosum Pall. Lignosum (Trautv.) Kitag 5. Ephedra sinica Stapf . 6. Glycyrrhiza uralensis Fisch. 7. Hedysarum scoparium Fisch.et Mey. 8. Artemisia sphaerocephala Krasch. 9. Caragana pygmaea (L.) DC. var. angustissima C. K. Schn. 10. Thermopsis lanceolata R.Br. 11. Astragalus melilotoides Pall. 14. Medicago sativa L.

Left part: a. Salix gordejevii Y. L. Chang et Skv. b. Caragana korshinskii Kom. c. Salix gordejevii Y. L. Chang et Skv. d. Hedysarum fruticosum Pall. var. mongolicum Turcz. ex B. Fedtsch. e. Calligonum mongolicum Turcz. f. Myricaria platyphylla Maxim. g. Medicago sativa L. h. Caragana intermedia Kuang. i. Hedysarum fruticosum Pall. var. lignosum (Trautv.) Kitag. j. Haloxylon a mmodendron (C. A. Mey.) Bunge. k. Alhagi sparsifolia Shap.

Acknowledgements. This work was supported by the Science and Technology Innovation Team (IRT 1259) of the Chinese Ministry of Education. Many thanks to professor Wang Liqun in Inner Mongolia Agricultural University for providing many valuable suggestions for this article! 


\section{REFERENCES}

[1] Achten, W. M. J., Verchot, L., Franken, Y. J., Harinder, P. S. M. (2008): Jatropha biodiesel production and use. - Biomass and Bioenergy 32(12): 1063-1084.

[2] Armstrong, W. P. (2001):. Modified roots, stems and leaves (Part 1). Botany 115, Vegetative Terminology (1). Available on: Http://waynesword.palomar.edu/.

[3] Canadell, J., Jackson, R. B., Ehleringer, J. R., Mooney, H. A., Sala, O. E., Schulze, E. D. (1996): Maximum rooting depth of vegetation types at the global scale - Oecologia 108: 583-595.

[4] Casper, B. B., Jackson, R. B. (1997): Plant competition underground. - Annual Review of Ecology and Systematics 28: 545-570.

[5] Christine, V. H., Brenda, B. C. (2002): Lateral root function and root overlap among mycorrhizal and nonmycorrhizal herbs in a Florida shrubland, measured using rubidium as a nutrient analog. - American Journal of Botany 89(8): 1289-1294.

[6] Douglas, B. K. (2011): Breeding crop plants with deep roots: their role in sustainable carbon, nutrient and water sequestration.- Annals of Botany 108: 407-418.

[7] Fu, C., Jiang, Z., Guan, Z., He, J., Xu, Z. (2008): Aridity Trend in Northern China. In: Fu, C., Jiang, Z., Guan, Z., He, J., Xu, Z. (eds). Regional Climate Studies of China. Springer Publishing House, New York.

[8] Göttlicher, S. G., Taylor, A. F. S,. Grip, H., Betson, N. R., Valinger, E., Högberg, M. N., Högberg, P. (2008): The lateral spread of tree root systems in boreal forests: Estimates based on ${ }^{15} \mathrm{~N}$ uptake and distribution of sporocarps of ectomycorrhizal fungi. - Forest Ecology and Management 255(1): 75-81.

[9] Gour, V. K. (2006): Production Practices Including Post-Harvest Management of Jatropha curcas. - In: Singh, B., Swaminathan, R., Ponraj, V. (eds.). Proceeding of the Biodiesel Conference Toward Energy Independence - Focus on Jatropha. New Delhi, India.

[10] Jackson, R. B., Sperry, J. S., Dawson, T. E. (2000): Root water uptake and transport: using physiological processes in global predictions. - Trends in Plant Science 5: 482-488.

[11] Jose, L. B., Alfredo, C. R., Luis, H. E. (2003): The role of nutrient availability in regulating root architecture. - Current Opinion in Plant Biology 6: 280-287.

[12] Liu, D. F., Chen, S. H. (2001): Root system types and reproductive characteristics of the plants on the northern grassland in China. - Proceedings (Paper collection) International Conference on Grassland Science and Industry. 242-245. (In Chinese with English abstract).

[13] Ma, W. H., Fang, J. Y. (2006): R:S ratios of temperate steppe and the environmental controls in Inner Mongolia. - Acta Scientiarum Naturalium Universitatis Pekinensis 42(6):774-778. (In Chinese with English abstract).

[14] Mokany, K., Raison, R., Prokushkin, A. (2006): Critical analysis of root:shoot ratios in terrestrial biomes. - Global Change Biology 12: 84-96.

[15] Neumann, R. B, Cardon, Z. G. (2012): The magnitude of hydraulic redistribution by plant roots: a review and synthesis of empirical and modeling studies. - New Phytologist 194: $337-352$

[16] Philippot, L., Hallin, S., Borjesson, G., Baggs, E. M. (2009): Biochemical cycling in the rhizosphere having an impact on global change. - Plant and Soil 321:61 - 81.

[17] Ruta, N., Liedgens, M., Fracheboud, Y., Peter, S., Hund, A. (2010): QTLs for the elongation of axile and lateral roots of maize in response to low water potential. Theoretical and Applied Genetics 120(3): 621-631.

[18] Schenk, H. J., Jackson, R B. (2002): Rooting depths, lateral root spreads, and belowground/aboveground allometries of plants in water limited ecosystems. - Journal of Ecology 90: 480-494.

[19] Smit, A. L, Bengough, A. G., Engels, C., van Noordwijk, M., Pellerin, S., van de Geijn, S. C. (2000): Root methods: a handbook (1st ed.). - Springer press, NewYork. 
[20] Wang, X. B.,Wu, P., Hu, B., Chen, Q. S. (2002): effects of Nitrate on the Growth of Lateral Root and Nitrogen Absorption in Rice. - Acta Botanica Sinica 44(6): 678-683.

[21] Waring, B. G., Powers, J. S. (2017): Overlooking what is underground: Root:shoot ratios and coarse root allometric equations for tropical forests - Forest Ecology and Management 385:10-15.

[22] Woo, Y. M., Park, H. J., Su'udi, M., Yang, J. I., Park, J. J., Back, K., Park, Y. M., An, G. (2007): Constitutively wilted 1, a member of the rice YUCCA gene family, is required for maintaining water homeostasis and an appropriate root to shoot ratio. - Plant Molecular Biology 65(1-2): 125-36.

[23] Yu, P., Li, X., White, P. J., Li, C . (2015): A Large and Deep Root System Underlies High Nitrogen-Use Efficiency in Maize Production. - PLoS ONE 10(5):1-17.

[24] Zamora, D. S., Jose, S., Nair, P. K. R. (2007): Morphological plasticity of cotton roots in response to interspecific competition with pecan in an alleycropping system in the southern United States. - Agroforestry Systems 69(2): 107-116.

[25] Zhang, H. L., Zhang, Q., Yue, P. (2016): Aridity over a semiarid zone in northern China and responses to the East Asian summer monsoon. - Journal Of Geophysical ResearchAtmospheres 121(23):13901-13918.

\section{Appendix}

Appendix 1. Rooting distribution by species with soil type or habitat

\begin{tabular}{|c|c|c|c|c|}
\hline Latin name & $\begin{array}{r}\text { Root } \\
\text { depth }\end{array}$ & $\begin{array}{c}\text { Lateral } \\
\text { root } \\
\text { spread }\end{array}$ & Root type & Soil and plant habitat \\
\hline Achillea millefolium $\mathrm{L}$. & 4.90 & 4.00 & Tap & Meadow chernozem \\
\hline $\begin{array}{l}\text { Achnatherum sibiricum (L.) } \\
\text { Keng }\end{array}$ & 28.20 & 32.40 & Fasciculate & Steppe \\
\hline $\begin{array}{l}\text { Achnatherum splendens } \\
\text { (Trin.) Nevski }\end{array}$ & 77.50 & 95.00 & Fasciculate & Meadow soil in Salinized soil \\
\hline $\begin{array}{l}\text { Achyrophorus ciliatus } \\
\text { (Thunb.) Makino }\end{array}$ & 21.23 & 17.31 & Tap & $\begin{array}{l}\text { Dark black soils in low wetland } \\
\text { between dunes }\end{array}$ \\
\hline Acorus calamus $\mathrm{L}$. & 45.28 & 121.70 & Rhizome & Bog soils in riverside \\
\hline $\begin{array}{l}\text { Adenophora gmelimii } \\
\text { (Spreng.) Fisch. }\end{array}$ & 40.00 & 1.88 & Tap & $\begin{array}{l}\text { The gaps between the rocks in } \\
\text { forest steppe }\end{array}$ \\
\hline $\begin{array}{c}\text { Adenophora gmelimii } \\
\text { (Spreng.) Fsch. var. } \\
\text { coronopifolia (Fisch.) Y. Z. } \\
\text { Zhao. }\end{array}$ & 24.67 & 4.00 & Tap & $\begin{array}{c}\text { Chernozems in shaded side of } \\
\text { hills }\end{array}$ \\
\hline $\begin{array}{l}\text { Adenophora stenanthina } \\
\text { (Ledeb.) Kitag. }\end{array}$ & 11.43 & 12.57 & Creeping & Aeolian soils \\
\hline $\begin{array}{l}\text { Adenophora tetrophylla } \\
\text { (Thunb.) Fisch. }\end{array}$ & 43.13 & 7.50 & Tap & Dark Kastanozems in ground \\
\hline $\begin{array}{c}\text { Adenophora trachelioides } \\
\text { Maxim. }\end{array}$ & 17.00 & 14.00 & Tap & Podzolic soils in hills \\
\hline $\begin{array}{l}\text { Agriophyllum squarrosum } \\
\text { (L.) Moq. }\end{array}$ & 16.86 & 10.00 & Tap & Sand in windward slope \\
\hline $\begin{array}{l}\text { Agriophyllum squarrosum } \\
\text { (L.) Moq. }\end{array}$ & 21.75 & 9.50 & Tap & Semi-fixed sand \\
\hline $\begin{array}{l}\text { Agriophyllum squarrosum } \\
\text { (L.) Moq. }\end{array}$ & 65.00 & 30.00 & Tap & Light brown calcic soils \\
\hline $\begin{array}{l}\text { Agropyron cristatum (L.) } \\
\text { Gaertn. }\end{array}$ & 39.86 & 13.29 & Fasciculate & Light Kastanozems \\
\hline Agropyron cristatum (L.) & 5.71 & 17.14 & Rhizome & Black soils in slope \\
\hline
\end{tabular}




\begin{tabular}{|c|c|c|c|c|}
\hline $\begin{array}{c}\text { Gaertn.var. pectiniforme } \\
\text { (Roem.et Schult.) H. L.Yang }\end{array}$ & & & & \\
\hline $\begin{array}{l}\text { Agropyron desertorum } \\
\text { (Fisch.) Schult. }\end{array}$ & 40.50 & 31.00 & Rhizome & Semi-fixed dune \\
\hline Agropyron michnoi Roshev. & 7.33 & 14.67 & Rhizome & Sandy Kastanozems \\
\hline Agropyron mongolicum Keng & 10.29 & 10.29 & Rhizome & Aeolian soils in meadow steppe \\
\hline Agropyron mongolicum Keng & 33.14 & 18.29 & Rhizome & Fixed dune \\
\hline Agropyron mongolicum Keng & 33.14 & 18.29 & Rhizome & $\begin{array}{l}\text { Light Kastanozems in overlying } \\
\text { sand }\end{array}$ \\
\hline $\begin{array}{c}\text { Agropyron mongolicum Keng } \\
\text { var. villosum H. .L. Yang }\end{array}$ & 6.56 & 6.44 & Rhizome & Sandy steppe \\
\hline Agrostis clavata Trin. & 5.00 & 6.00 & Rhizome & Meadow \\
\hline Agrostis divaricatissima $\mathrm{Mez}$ & 5.33 & 4.17 & Rhizome & Meadow in lowland flood plain \\
\hline Agrostis gigantea Roth & 4.86 & 6.57 & Rhizome & Meadow in lowland flood plain \\
\hline $\begin{array}{c}\text { Ajania achilleoides (Turcz.) } \\
\text { Poljak. ex Grub. }\end{array}$ & 7.64 & 3.27 & Tap & Stony brown calcic soils \\
\hline $\begin{array}{c}\text { Ajania achilleoides (Turcz.) } \\
\text { Poljak. ex Grub. }\end{array}$ & 19.27 & 11.82 & Tap & Gravel sandy soil \\
\hline $\begin{array}{c}\text { Aleuritopteris argentea } \\
\text { (Gme'1.) Fe'e }\end{array}$ & 4.44 & 3.00 & Fibrous & Under the shrub in shade \\
\hline Alhagi sparsifolia Shap. & 21.25 & 6.75 & Tap & Semi-fixed dune \\
\hline Alhagi sparsifolia Shap. & 39.00 & 25.00 & Tap & Gray-brown desert soil \\
\hline Alhagi sparsifolia Shap. & 85.00 & 170.00 & Tap & Aeolian soils \\
\hline Alhagi sparsifolia Shap. & 155.00 & 75.00 & Tap & Desert \\
\hline Alhagi sparsifolia Shap. & $\begin{array}{c}1200.0 \\
0\end{array}$ & 600.00 & Tap & Desert ( one year old) \\
\hline $\begin{array}{l}\text { Alisma orientale (G. Sam.) } \\
\text { Juz. }\end{array}$ & 28.06 & 57.10 & Tuber & In river \\
\hline Allium condensatum Turcz. & 33.90 & 18.00 & Tuber & Litho soils in slope \\
\hline Allium mongolicum Regel. & 5.67 & 4.17 & Tuber & Desert steppe \\
\hline $\begin{array}{l}\text { Allium polyrhizum Turcz. ex } \\
\text { Regel }\end{array}$ & 19.04 & 8.09 & Tuber & $\begin{array}{l}\text { Brown calcic soils in desert } \\
\text { steppe }\end{array}$ \\
\hline Allium ramosum $\mathrm{L}$. & 37.14 & 31.43 & Tuber & $\begin{array}{l}\text { Litho soils in hills of meadow } \\
\text { steppe }\end{array}$ \\
\hline Allium senescens $\mathrm{L}$ & 9.14 & 5.43 & Tuber & Light Kastanozems in hills \\
\hline Allium senescens $\mathrm{L}$ & 14.20 & 12.60 & Tuber & Dark Kastanozems in hills \\
\hline Allium tenuissimum L. & 11.20 & 9.20 & Tuber & Steppe and sandy steppe \\
\hline $\begin{array}{c}\text { Alopecurus brachystachyus } \\
\text { Marsh. -Bieb. }\end{array}$ & 28.00 & 22.00 & Rhizome & Meadow in lowland flood plain \\
\hline Amaranthus retroflexus L. & 26.63 & 7.50 & Tap & Kastanozems \\
\hline $\begin{array}{c}\text { Anabasis brevifolia C.A. } \\
\text { Mey. }\end{array}$ & 48.00 & 35.50 & Tap & Brown sandy soil in ground \\
\hline $\begin{array}{c}\text { Anabasis brevifolia C.A. } \\
\text { Mey. }\end{array}$ & 52.32 & 20.63 & Tap & $\begin{array}{l}\text { Light Kastanozems in ground } \\
\text { between dunes }\end{array}$ \\
\hline Androsace longifolia Turcz. & 21.20 & 12.60 & Tap & $\begin{array}{c}\text { Dark Kastanozems between } \\
\text { dunes }\end{array}$ \\
\hline Androsace mariae Kanitz & 5.04 & 4.32 & Tap & Skeletol soils in hills \\
\hline $\begin{array}{l}\text { Anemarrhena asphodeloides } \\
\text { Bunge }\end{array}$ & 20.20 & 13.40 & Fibrous & Meadow steppe \\
\hline Arctium lappa L. & 23.57 & 9.14 & Tap & Forest steppe in hills \\
\hline Arenaria juncea Bieb. & 5.55 & 3.84 & Tap & Light Kastanozems in hills \\
\hline Aristida adscenionis L. & 7.50 & 5.50 & Fasciculate & $\begin{array}{l}\text { Brown calcic soils in lowland of } \\
\text { desert steppe }\end{array}$ \\
\hline $\begin{array}{l}\text { Artemisia anethifolia Web. ex } \\
\text { Stechm. }\end{array}$ & 28.91 & 7.64 & Tap & Saline and Salinized soil \\
\hline Artemisia annua L. & 18.22 & 6.44 & Tap & Brown earth soil in hills \\
\hline
\end{tabular}




\begin{tabular}{|c|c|c|c|c|}
\hline Artemisia апnиa $\mathrm{L}$. & 18.46 & 6.92 & Tap & Wetland of riverside in hills \\
\hline Artemisia anпua $\mathrm{L}$. & 32.00 & 25.60 & Tap & Light Kastanozems in hills \\
\hline Artemisia annua L. & 54.17 & 10.42 & Tap & Brown earth aeolian soil \\
\hline $\begin{array}{l}\text { Artemisia blepharolepis } \\
\text { Bunge }\end{array}$ & 33.50 & 17.50 & Tap & Fixed sand \\
\hline Artemisia brachyloba Franch. & 65.49 & 87.68 & Tap & Mountain \\
\hline Artemisia desertorum Spreng. & 94.80 & 13.20 & Tap & Kastanozems in hills \\
\hline Artemisia dracunculus Linn. & 7.20 & 9.90 & Tap & Light Kastanozems \\
\hline Artemisia frigida Willd. & 23 & 13.75 & Tap & $\begin{array}{l}\text { Sandy Kastanozems-in } \\
\text { vegetation growth stage }\end{array}$ \\
\hline Artemisia frigida Willd. & 99 & 13.5 & Tap & $\begin{array}{l}\text { Sandy Kastanozems-in } \\
\text { reproductive stage }\end{array}$ \\
\hline $\begin{array}{l}\text { Artemisia halodendron Turcz. } \\
\text { ex Bess. }\end{array}$ & 158.47 & 35.08 & Tap & Semi-mobile sand \\
\hline $\begin{array}{c}\text { Artemisia lavandulaefolia D. } \\
\text { C. }\end{array}$ & 104.12 & 68.82 & Creeping & Sand \\
\hline $\begin{array}{c}\text { Artemisia mongolica (Fisch. } \\
\text { ex Bess.) Nakai. }\end{array}$ & 6.58 & 5.25 & Creeping & Kastanozems in meadow \\
\hline Artemisia ordosica Krasch. & 25.83 & 21.67 & Tap & Sandy brown calcic soils \\
\hline Artemisia ordosica Krasch. & 117.14 & 78.57 & Tap & Semi-fixed sand \\
\hline Artemisia oxycephala Kitag. & 37.50 & 43.75 & Tap & $\begin{array}{l}\text { Semi-fixed dune in meadow } \\
\text { steppe }\end{array}$ \\
\hline Artemisia palustris L. & 31.38 & 16.00 & Tap & Sand in forest steppe \\
\hline Artemisia pubescens Ledeb. & 13.80 & 6.00 & Tap & Dry riverbed alluvial soils \\
\hline Artemisia sacrorum Ledeb. & 22.33 & 17.33 & Creeping & Dark Kastanozems in hills \\
\hline $\begin{array}{l}\text { Artemisia sacrorum Ledeb. } \\
\text { Var. incana (Barr.) Y. R. Ling }\end{array}$ & 29.10 & 19.20 & Creeping & Sandy dark Kastanozems \\
\hline Artemisia sieversiana & 35.00 & 25.00 & Tap & $\begin{array}{l}\text { Kastanozems in dump of coal } \\
\text { mine in steppe }\end{array}$ \\
\hline $\begin{array}{c}\text { Artemisia sphaerocephala } \\
\text { Krasch. }\end{array}$ & 74.48 & 122.07 & Tap & Semi-mobile sand \\
\hline $\begin{array}{c}\text { Artemisia sphaerocephala } \\
\text { Krasch. }\end{array}$ & 171.43 & 100.00 & Tap & Dune \\
\hline Artemisia subulata Nakai & 16.89 & 14.89 & Creeping & Alluvial soils in dry riverbed \\
\hline Artemisia tanacetifolia $\mathrm{L}$. & 14.70 & 15.90 & Creeping & Forest steppe \\
\hline Artemisia xerophytica Krasch. & 60.00 & 37.24 & Tap & $\begin{array}{l}\text { Light brown calcic soils in slope } \\
\text { eroded by wind }\end{array}$ \\
\hline Artemisia xerophytica Krasch. & 73.45 & 40.34 & Tap & $\begin{array}{l}\text { Light brown calcic soils in high } \\
\text { plains }\end{array}$ \\
\hline Arundinella anomala Steud. & 24.00 & 23.50 & Rhizome & Aeolian soils \\
\hline $\begin{array}{c}\text { Asparagus dauricus Fisch.ex } \\
\text { Link }\end{array}$ & 40.83 & 17.50 & Fibrous & Semi-fixed dune \\
\hline $\begin{array}{c}\text { Asparagus gobicus Ivan.ex } \\
\text { Grub. }\end{array}$ & 116.84 & 82.11 & Fibrous & Stony steppe desert \\
\hline $\begin{array}{c}\text { Asparagus oligoclonos } \\
\text { Maxim. }\end{array}$ & 12.00 & 7.56 & Fibrous & Litho soils in hills \\
\hline Aster alpinus L. & 21.67 & 18.00 & Tap & Litho soils in hills \\
\hline $\begin{array}{l}\text { Asterothamnus centrali- } \\
\text { asiaticus Novopokr. }\end{array}$ & 23.00 & 9.00 & Tap & $\begin{array}{l}\text { Gravelly desert soil in desert } \\
\text { steppe }\end{array}$ \\
\hline $\begin{array}{l}\text { Asterothamnus centrali- } \\
\text { asiaticus Novopokr. }\end{array}$ & 78.75 & 75.00 & Tap & $\begin{array}{l}\text { Brown calcic soils in desert } \\
\text { steppe }\end{array}$ \\
\hline Astragalus adsurgens Pall. & 23.14 & 11.14 & Tap & Fluvo-aquic soils between dunes \\
\hline Astragalus adsurgens Pall. & 55.45 & 43.64 & Tap & Humid sandy soil \\
\hline Astragalus adsurgens Pall. & 205.33 & 32.00 & Tap & $\begin{array}{c}\text { Light and stony Kastanozems in } \\
\text { slope }\end{array}$ \\
\hline Astragalus complanatus & 64.90 & 12.02 & Tap & Meadow steppe and alkalization \\
\hline
\end{tabular}




\begin{tabular}{|c|c|c|c|c|}
\hline Bunge & & & & meadow \\
\hline $\begin{array}{c}\text { Astragalus efoliolatus Hand.- } \\
\text { Mazz. }\end{array}$ & 38.75 & 10.31 & Tap & Desert steppe \\
\hline Astragalus galactites Pall. & 25.05 & 5.25 & Tap & Light brown calcic soils \\
\hline Astragalus galactites Pall. & 40.53 & 10.00 & Tap & Light Kastanozems \\
\hline Astragalus galactites Pall. & 106.97 & 21.52 & Tap & Gravelly brown soil \\
\hline Astragalus melilotoides Pall. & 336.99 & 24.66 & Tap & Fixed sand \\
\hline Astragalus miniatus Bunge & 16.55 & 10.55 & Tap & $\begin{array}{c}\text { Stony slope and Salinized } \\
\text { lowland }\end{array}$ \\
\hline $\begin{array}{c}\text { Astragalus scaberrimus } \\
\text { Bunge } \\
\end{array}$ & 53.89 & 28.33 & Creeping & Kastanozems in stony slope \\
\hline $\begin{array}{l}\text { Atraphaxis frutescens (L.) } \\
\text { Ewersm. }\end{array}$ & 78.33 & 200.00 & Tap & Flat sand \\
\hline $\begin{array}{l}\text { Atraphaxis frutescens (L.) } \\
\text { Ewersm. }\end{array}$ & 160.00 & 40.00 & Tap & Sand in high plains \\
\hline Atraphaxis manshurica Kitag. & 156.65 & 41.77 & Tap & Sand in plain \\
\hline $\begin{array}{c}\text { Atraphaxis pungens (Bieb.) } \\
\text { Jaub. et Spach. }\end{array}$ & 95.00 & No data & Tap & Sandy chestnut soil \\
\hline Atriplex sibirica $\mathrm{L}$. & 63.00 & 11.50 & Tap & Salinized lowland \\
\hline $\begin{array}{c}\text { Bassia dasyphylla (Fsch.et } \\
\text { Mey.). Kuntze }\end{array}$ & 8.36 & 2.91 & Tap & Fixed sand \\
\hline $\begin{array}{c}\text { Bassia dasyphylla (Fsch.et } \\
\text { Mey.). Kuntze }\end{array}$ & 16.17 & 11.67 & Tap & Desert soil \\
\hline $\begin{array}{l}\text { Bassia dasyphylla (Fsch.et } \\
\text { Mey.). Kuntze }\end{array}$ & 31.13 & 25.50 & Tap & Kastanozems in slope \\
\hline $\begin{array}{l}\text { Beckmannia syzigachne } \\
\text { (Steud.) Fernald }\end{array}$ & 7.69 & 2.23 & Fasciculate & Swampy meadow and meadow \\
\hline Blysmus rufus (Huds.) Link & 6.20 & 7.80 & Rhizome & Meadow soil in low wetland \\
\hline $\begin{array}{l}\text { Bothriochloa ischamum (L.) } \\
\text { Keng }\end{array}$ & 19.13 & 17.25 & Rhizome & Skeletol soils in hills \\
\hline $\begin{array}{c}\text { Bromus ciliatus L. var. } \\
\text { richardsonii (Link) Y.Q. } \\
\text { Jiang }\end{array}$ & 34.00 & 29.00 & Rhizome & Dark Kastanozems \\
\hline Bromus inermis Leyss. & 21.75 & 58.50 & Rhizome & $\begin{array}{l}\text { Dark Kastanozems in hills of } \\
\text { typical steppe }\end{array}$ \\
\hline Bupleurum bicaule Helm & 22.00 & 4.44 & Tap & Dark Kastanozems \\
\hline Bupleurum chinense D. C. & 13.78 & 2.78 & Tap & Kastanozems in hills \\
\hline $\begin{array}{c}\text { Bupleurum scorzonerifolium } \\
\text { Willd. }\end{array}$ & 29.60 & 6.40 & Tap & Light Kastanozems in hills \\
\hline Butomus umbellatus L. & 17.86 & 20.71 & Fibrous & Bog soils in riverside \\
\hline $\begin{array}{l}\text { Calamagrostis epigeios (L.) } \\
\text { Roth }\end{array}$ & 55.00 & 51.00 & Rhizome & Meadow soil in salinized ground \\
\hline $\begin{array}{c}\text { Calamagrostis } \\
\text { pseudophragmites (Hall.f) } \\
\text { Koel }\end{array}$ & 21.50 & 46.50 & Rhizome & Fixed dune \\
\hline $\begin{array}{c}\text { Calligonum mongolicum } \\
\text { Turcz. }\end{array}$ & 162.00 & 372.00 & Tap & Desert steppe sand \\
\hline $\begin{array}{l}\text { Calystegia haderacea Wall. } \\
\text { Ex Roxb. }\end{array}$ & 13.56 & 10.22 & Creeping & Aeolian soil \\
\hline Caragana intermedia Kuang. & 620.00 & 142.11 & Tap & Dry riverbed in desert steppe \\
\hline Caragana korshinskii Kom. & 262.77 & 1576.6 & Tap & Aeolian soil \\
\hline Caragana microphylla Lam. & 363.64 & 79.55 & Tap & $\begin{array}{l}\text { Light Kastanozems in hills( } \\
\text { typical steppe ) }\end{array}$ \\
\hline Caragana microphylla Lam. & 388.89 & 38.89 & Tap & $\begin{array}{c}\text { Light Kastanozems in slope of } \\
\text { hills(desert steppe) }\end{array}$ \\
\hline Caragana microphylla Lam. & 160.00 & 43.84 & Tap & Typical steppe \\
\hline
\end{tabular}




\begin{tabular}{|c|c|c|c|c|}
\hline Var. cinerea Kom. & & & & \\
\hline $\begin{array}{l}\text { Caragana pygmaea (L.) D. C. } \\
\text { var. angustissima C. K. Schn. }\end{array}$ & 252.71 & 80.62 & Tap & $\begin{array}{l}\text { Light Kastanozems in slope of } \\
\text { hills(Desert steppe) }\end{array}$ \\
\hline Caragana stenophylla Pojark. & 172.17 & 36.52 & Creeping & Sandy soil in desert steppe \\
\hline Caragana tibetica Kom. & 133.75 & 25.00 & Tap & Steppe Desert \\
\hline Carex duriuscula C. A. Mey. & 7.89 & 15.79 & Rhizome & Fixed sand \\
\hline Carex duriuscula C. A. Mey. & 97.06 & 79.41 & Rhizome & $\begin{array}{l}\text { Light Kastanozems of gentle } \\
\text { slope in hills }\end{array}$ \\
\hline Carex gotoi Ohwi. & 3.67 & 3.67 & Rhizome & Meadow soil in between dunes \\
\hline Carex gotoi Ohwi. & 5.44 & 14.25 & Rhizome & Meadow soil in wetland \\
\hline Carex kobomugi Ohwi. & 14.67 & 23.33 & Rhizome & Riverside sandy soil \\
\hline Carex lithophila Turcz. & 6.25 & 1.25 & Rhizome & Meadow soil in forest steppe \\
\hline Carex raddei kukenth. & 54.00 & 69.00 & Rhizome & Meadow soil in valley \\
\hline $\begin{array}{l}\text { Carex rigescens (Franch.) V. } \\
\text { Krecz. }\end{array}$ & 18.86 & 16.57 & Rhizome & $\begin{array}{c}\text { Sandybrown calcic soils in } \\
\text { typical steppe }\end{array}$ \\
\hline $\begin{array}{l}\text { Carex stenophylloides V. } \\
\text { Krecz. }\end{array}$ & 12.25 & 16.50 & Rhizome & Bog soils in riverside \\
\hline $\begin{array}{l}\text { Carex stenophylloides V. } \\
\text { Krecz. }\end{array}$ & 35.00 & 36.50 & Rhizome & $\begin{array}{l}\text { Fluvo-aquic soils in lowland of } \\
\text { Hills }\end{array}$ \\
\hline $\begin{array}{c}\text { CaryopteriS mongholica } \\
\text { Bunge }\end{array}$ & 117.27 & 169.09 & Tap & $\begin{array}{l}\text { Light Kastanozems between } \\
\text { dunes }\end{array}$ \\
\hline $\begin{array}{c}\text { Centaurtum pulchellum } \\
\text { (Swartz) Druce var. altacum } \\
\text { (Griseb.) Krtag. }\end{array}$ & 9.56 & 1.78 & Tap & $\begin{array}{l}\text { Meadow between dunes in } \\
\text { mobile sand }\end{array}$ \\
\hline $\begin{array}{c}\text { Ceratoides arborescens } \\
\text { (Losinsk.) Tsien et C. G. Ma }\end{array}$ & 187.69 & 98.46 & Tap & Light Kastanozems in meadow \\
\hline $\begin{array}{c}\text { Ceratoides lateens (J. F. } \\
\text { Gmel.) Reveal et Holmgren. }\end{array}$ & 61.11 & 64.44 & Tap & $\begin{array}{c}\text { Brown calcic soils in steppe } \\
\text { desert }\end{array}$ \\
\hline $\begin{array}{c}\text { Ceratoides lateens (J. F. } \\
\text { Gmel.) Reveal et Holmgren. }\end{array}$ & 84.55 & 31.36 & Tap & $\begin{array}{l}\text { Light Kastanozems in hills of } \\
\text { steppe desert }\end{array}$ \\
\hline $\begin{array}{l}\text { Chamaenerion angustifolium } \\
\text { (L.) Scop. }\end{array}$ & 14.67 & 16.67 & Tap & Black soils in hills \\
\hline $\begin{array}{l}\text { Chamaerhodos altaica } \\
\text { (Laxm.) Bge. }\end{array}$ & 45.00 & 32.63 & Tap & Litho soils in hills \\
\hline $\begin{array}{c}\text { Chamaerhodos canescens } \\
\text { Krause }\end{array}$ & 16.00 & 9.33 & Tap & Dark Kastanozems in hills \\
\hline $\begin{array}{c}\text { Chamaerhodos erecta (L.) } \\
\text { Bge. }\end{array}$ & 32.00 & 6.29 & Tap & Sandy brown soil in slope \\
\hline $\begin{array}{c}\text { Chamaerhodos erecta (L.) } \\
\text { Bge. }\end{array}$ & 45.43 & 12.43 & Tap & Stony Kastanozems in slope \\
\hline Chamaerhodos trifida Ldb. & 21.20 & 5.60 & Tap & Litho soils in hills \\
\hline Chenopodium album $\mathrm{L}$. & 10.00 & 5.00 & Tap & Mesophytic weed \\
\hline $\begin{array}{l}\text { Chenopodium acuminatum } \\
\text { Willd. }\end{array}$ & 9.30 & 6.60 & Tap & $\begin{array}{l}\text { Light Salinized soil in low } \\
\text { wetland }\end{array}$ \\
\hline $\begin{array}{l}\text { Chenopodium acuminatum } \\
\text { Willd. } \\
\end{array}$ & 13.83 & 9.33 & Tap & Sandy Kastanozems in hills \\
\hline Chenopodium aristatum L. & 48.21 & 22.14 & Tap & Fixed sand \\
\hline Chenopodium glaucum L. & 5.92 & 5.67 & Tap & Sand between dunes \\
\hline Chloris virgata Swartz. & 20.00 & 27.78 & Fasciculate & Fluvo-aquic soils in lowland \\
\hline Chloris virgata Swartz. & 26.67 & 14.67 & Fasciculate & Light brown calcic soils \\
\hline $\begin{array}{l}\text { Cirsium segetum (Willd.) M } \\
\text { B. }\end{array}$ & 10.00 & 8.17 & Creeping & Alluvial soils \\
\hline $\begin{array}{c}\text { Cistanche salsa (C. A. Mey) } \\
\text { G. Beck. }\end{array}$ & 38.73 & 19.91 & Fibrous & $\begin{array}{c}\text { Saline-alkali soil in lake basin of } \\
\text { desert steppe }\end{array}$ \\
\hline Cleistogenes polyphylla Keng. & 7.30 & 4.40 & Fasciculate & $\begin{array}{l}\text { Stony Kastanozems in typical } \\
\text { steppe }\end{array}$ \\
\hline
\end{tabular}




\begin{tabular}{|c|c|c|c|c|}
\hline Cleistogenes polyphylla Keng. & 12.00 & 4.50 & Fasciculate & Aeolian sand in hills \\
\hline $\begin{array}{l}\text { Cleistogenes songorica } \\
\text { (Roshev.) Ohwi. }\end{array}$ & 23.11 & 9.78 & Fasciculate & $\begin{array}{l}\text { Light brown calcic soils in desert } \\
\text { steppe }\end{array}$ \\
\hline $\begin{array}{l}\text { Cleistogenes squarrosa } \\
\text { (Trin.) Keng. }\end{array}$ & 10.13 & 7.25 & Fasciculate & $\begin{array}{l}\text { Sandy Kastanozems in typical } \\
\text { steppe }\end{array}$ \\
\hline Clematis aethusifolia Turcz. & 38.33 & 11.11 & Tap & Sandy light Kastanozems \\
\hline $\begin{array}{l}\text { Clematis canescens (Turcz.) } \\
\text { W. T. Wanget M.C. Chang. }\end{array}$ & 49.44 & 20.00 & Tap & Fixed sand \\
\hline Clematis hexapetala Pall. & 43.13 & 20.00 & Tap & Dark meadow soil in valley \\
\hline $\begin{array}{l}\text { Cnidium dahuricum (Jacq.) } \\
\text { Turcz. ex Fisch. et Mey. }\end{array}$ & 5.58 & 2.00 & Tap & Light salined meadow soil \\
\hline $\begin{array}{l}\text { Codonopsis pilosula (Franch.) } \\
\text { Nannf. } \\
\end{array}$ & 18.57 & 10.86 & Tap & Shrub in forest edge of hills \\
\hline Convolvulus ammannii Desr. & 12.52 & 3.52 & Tap & Sandy light Kastanozems \\
\hline Convolvulus ammannii Desr. & 18.58 & 2.71 & Tap & Saline soil in meadow \\
\hline Convolvulus ammannii Desr. & 36.57 & 6.57 & Tap & Light brown calcic soils in hills \\
\hline Convolvulus ammannii Desr. & 55.56 & 10.00 & Tap & Brown calcic soils \\
\hline Convolvulus arvensis L. & 9.25 & 8.50 & Creeping & Light Kastanozems \\
\hline Corispenmum puberulum lljin & 46.64 & 14.73 & Tap & Sand in steppe \\
\hline $\begin{array}{l}\text { Corispermum mongolicum } \\
\text { Iljin. }\end{array}$ & 21.20 & 10.40 & Tap & Sandy Kastanozems in hills \\
\hline $\begin{array}{l}\text { Corsipermum declinatum var. } \\
\text { tylocarp Um (Hance) Tsien et } \\
\text { C.G. Ma. }\end{array}$ & 24.50 & 11.00 & Tap & Semi-fixed sand \\
\hline $\begin{array}{l}\text { Corsipermum declinatum var. } \\
\text { tylocarp Um (Hance) Tsien et } \\
\text { C. G. Ma. }\end{array}$ & 45.00 & 13.13 & Tap & Light brown calcic soils \\
\hline $\begin{array}{c}\text { Crepis crocea (Lam.) } \\
\text { Babcock. }\end{array}$ & 51.00 & 28.50 & Creeping & Skeleton chernozems \\
\hline Crypsis aculeata (L.) Art. & 10.13 & 9.47 & Fasciculate & $\begin{array}{c}\text { SalinizedSalinized meadow or } \\
\text { humid saline in riverside of } \\
\text { desert steppe }\end{array}$ \\
\hline Cymbaria dahurica $\mathrm{L}$. & 10.00 & 29.00 & Tap & Salinized Kastanozems \\
\hline Cymbaria dahurica $\mathrm{L}$. & 21.33 & 24.67 & Tap & Light Kastanozems \\
\hline Cynanchum chinense $\mathrm{R}$. Br. & 61.00 & 31.90 & Creeping & Salinized sand \\
\hline $\begin{array}{l}\text { Cynanchum komarovii Al. } \\
\text { Iljinski. }\end{array}$ & 29.20 & 9.60 & Fibrous & Sand( two years old) \\
\hline $\begin{array}{l}\text { Cynanchum thesioides } \\
\text { (Freyn) K. Schum. }\end{array}$ & 70.00 & 120.00 & Creeping & Semi-fixed sand \\
\hline $\begin{array}{c}\text { Cynomorium songaricum } \\
\text { Rupr. }\end{array}$ & 31.14 & 14.86 & Fibrous & Lowland between dunes in desert \\
\hline Datura metel L. & 16.40 & 13.00 & Tap & Kastanozems \\
\hline Delphinium grandiflorum L. & 4.00 & 3.78 & Tap & Dark Kastanozems in hills \\
\hline $\begin{array}{l}\text { Dendranthema zawadskii } \\
\text { (Herb.) Tzvel. }\end{array}$ & 15.60 & 15.00 & Creeping & Skeleton dark Kastanozems \\
\hline $\begin{array}{l}\text { Deyeuxia langsdorffii (Link) } \\
\text { Kunth. }\end{array}$ & 31.25 & 21.25 & Rhizome & Dark Kastanozems \\
\hline $\begin{array}{l}\text { Dgitaria ischaemum (Schreb.) } \\
\text { Schreb. }\end{array}$ & 13.27 & 11.45 & Fasciculate & Humid areas and sandy \\
\hline $\begin{array}{l}\text { Dianthus chinensis L.var. } \\
\text { subulifolius (kitag) Y. c.Ma. }\end{array}$ & 34.33 & 6.67 & Tap & Skeletol soils in hills \\
\hline Dianthus chinesis L. & 25.33 & 2.67 & Tap & Skeletol soils in hills \\
\hline Dictamnus dasycarpus Turcz. & 19.50 & 10.50 & Tap & Chernozems \\
\hline $\begin{array}{l}\text { Dontostemon crassifolius } \\
\text { (Bunge) Maxim. }\end{array}$ & 28.24 & 44.12 & Creeping & $\begin{array}{l}\text { Light brown calcic soils in } \\
\text { overlying sand }\end{array}$ \\
\hline Dontostemon eglandulosus & 5.80 & 1.80 & Tap & Light Kastanozems in hills \\
\hline
\end{tabular}




\begin{tabular}{|c|c|c|c|c|}
\hline (D. C.) Ledeb. & & & & \\
\hline $\begin{array}{c}\text { Dontostemon integrifolius (L.) } \\
\text { Ledeb. }\end{array}$ & 14.00 & 20.67 & Creeping & Light Kastanozems \\
\hline $\begin{array}{c}\text { Dracocephalum } \\
\text { heterophyllum Benth. }\end{array}$ & 51.50 & 7.50 & Tap & $\begin{array}{l}\text { Light Kastanozems in ground of } \\
\text { hills }\end{array}$ \\
\hline Dracocephalum moldavica $\mathrm{L}$. & 29.63 & 12.75 & Tap & $\begin{array}{c}\text { Gravel and sandy brown calcic } \\
\text { soils in slope }\end{array}$ \\
\hline Dracocephalum moldavica L. & 96.67 & 25.56 & Tap & Fixed sand \\
\hline $\begin{array}{c}\text { Echinochloa crusgalli (L.) } \\
\text { Beauv. }\end{array}$ & 17.50 & 10.50 & Fasciculate & Humid areas \\
\hline Echinops gmelini Turcz. & 17.67 & 4.00 & Tap & $\begin{array}{l}\text { Sandy Kastanozems in high } \\
\text { plains }\end{array}$ \\
\hline Echinops gmelini Turcz. & 19.00 & 2.67 & Tap & Wet saline soil \\
\hline Eleocharis intersita Zinserl. & 9.60 & 7.60 & Rhizome & Wetlands in riverside \\
\hline Elymus dahuricus Turcz. & 53.33 & 33.33 & Fasciculate & $\begin{array}{l}\text { Meadow or mountain meadow } \\
\text { steppe }\end{array}$ \\
\hline Elymus excelsus Turcz. & 10.09 & 4.36 & Fasciculate & $\begin{array}{l}\text { Mountain meadow or meadow } \\
\text { steppe }\end{array}$ \\
\hline $\begin{array}{c}\text { Elymus purpuraristatus C. P. } \\
\text { Wang et H. L. Yang. }\end{array}$ & 7.75 & 5.00 & Rhizome & Chernozems \\
\hline Elymus sibiricus L. & 8.67 & 4.25 & Fasciculate & Meadow or meadow steppe \\
\hline $\begin{array}{l}\text { Enneapogon borealis } \\
\text { (Griseb.) Honda. }\end{array}$ & 6.22 & 3.33 & Fasciculate & Desert and Desert steppe \\
\hline Ephedra przewalskii Stapf. & 125.77 & 73.85 & Creeping & Sand \\
\hline Ephedra sinica Stapf. & 53.75 & 225.00 & Creeping & Sandy soil in fixed sand \\
\hline Equisetum fluviatile L. & 32.57 & 7.71 & Rhizome & $\begin{array}{l}\text { Swampy or swampy grassland in } \\
\text { forest steppe }\end{array}$ \\
\hline Equisetum pratense Ehrh. & 9.80 & 12.00 & Rhizome & Sandy meadow soil in wetland \\
\hline Equisetum sylvaticum L. & 10.63 & 7.25 & Rhizome & Low-lying land in riverside \\
\hline Eragrostis minor Host. & 24.67 & 26.67 & Fasciculate & $\begin{array}{c}\text { Sandy brown calcic soils in slope } \\
\text { of hills }\end{array}$ \\
\hline Eragrostis pilosa (L.) Beauv. & 4.11 & 1.67 & Fasciculate & Meadow \\
\hline $\begin{array}{l}\text { Eragrostis pilosa (L.) Beauv. } \\
\text { var. imbenbis } \text { Franch. }\end{array}$ & 19.33 & 20.00 & Fasciculate & Semi-fixed sand \\
\hline Erodium stephanianum Willd. & 142.50 & 12.50 & Tap & Low wetland in riverside \\
\hline Euphorbia esula L. & 17.20 & 1.90 & Creeping & Brown earth types of sandy soil \\
\hline Euphorbia esula L. & 36.00 & 34.29 & Creeping & Fixed sand \\
\hline Euphorbia esula L. & 61.50 & 93.00 & Creeping & Semi-fixed sand \\
\hline Euphorbia esula L. & 73.60 & 16.40 & Creeping & Kastanozems \\
\hline Euphorbia fischeriana Steud. & 30.50 & 25.00 & Tap & Dark Kastanozems \\
\hline $\begin{array}{c}\text { Euphorbia humifusa Willd. ex } \\
\text { Schlecht. }\end{array}$ & 5.00 & 3.75 & Tap & Aeolian sand in slope \\
\hline $\begin{array}{l}\text { Euphorbia humifusa Willd. ex } \\
\text { Schlecht. }\end{array}$ & 16.25 & 5.63 & Tap & Aeolian soils in flat groud \\
\hline Ferula bungeana Kitag. & 137.42 & 13.55 & Tap & Aeolian soil \\
\hline Festuca ovina L. & 7.00 & 5.09 & Fasciculate & Dark Kastanozems \\
\hline Festuca rubra L. & 7.63 & 7.00 & Rhizome & Dark Kastanozems in hills \\
\hline $\begin{array}{l}\text { Filifolium sibiricum (L.) } \\
\text { Kitam. }\end{array}$ & 24.75 & 18.50 & Tap & Litho soils in hills \\
\hline $\begin{array}{l}\text { Filifolium sibiricum (L.) } \\
\text { Kitam. }\end{array}$ & 43.00 & 19.00 & Tap & Kastanozems in hills \\
\hline Galium verum $\mathrm{L}$. & 61.71 & 16.00 & Tap & $\begin{array}{c}\text { Loamy and sandy typical } \\
\text { Kastanozems }\end{array}$ \\
\hline $\begin{array}{c}\text { Garex pediformis C. A. } \\
\text {.Mey. }\end{array}$ & 12.00 & 5.43 & Rhizome & $\begin{array}{c}\text { Dark Kastanozems of sunny } \\
\text { slope in hills } \\
\text { （Meadow steppe }\end{array}$ \\
\hline
\end{tabular}




\begin{tabular}{|c|c|c|c|c|}
\hline Gentiana dahurica Fisch. & 51.00 & 15.00 & Tap & Light Kastanozems in high plains \\
\hline Gentiana macrophylla Pall. & 16.57 & 6.57 & Tap & Meadow soil in riverside \\
\hline Geranium sibiricum L. & 22.78 & 21.67 & Fibrous & Meadow soil in riverside \\
\hline Glaux maritime $\mathrm{L}$. & 20.20 & 8.40 & Fibrous & $\begin{array}{l}\text { Bog soils in fluvial sand of } \\
\text { riverside }\end{array}$ \\
\hline Glycine soja Sieb. et Zucc. & 12.29 & 10.00 & Tap & Flood plain \\
\hline Glycyrrhiza uralensis Fisch. & 97.50 & 223.50 & Creeping & Typical steppe \\
\hline $\begin{array}{l}\text { Goldbachia iaevigata (M. } \\
\text { Bieb.) D. C. }\end{array}$ & 50.00 & 13.18 & Tap & Light Kastanozems \\
\hline $\begin{array}{l}\text { Gueldenstaedtia gansuensis } \\
\text { H. P. Tsui. }\end{array}$ & 35.33 & 12.00 & Tap & Semi-fixed dune \\
\hline $\begin{array}{c}\text { Gueldenstaedtia stenophylla } \\
\text { Bunge }\end{array}$ & 37.00 & 3.75 & Tap & Sandy steppe \\
\hline $\begin{array}{c}\text { Gymnadenia conopsea (L.) R. } \\
\text { Br. }\end{array}$ & 15.20 & 11.20 & Tuber & $\begin{array}{l}\text { Dark Kastanozems in low } \\
\text { wetland }\end{array}$ \\
\hline Gypsophila licentiana-Mazz. & 25.14 & 11.43 & Tap & Litho soils in hills \\
\hline $\begin{array}{l}\text { Gysophila desertorum } \\
\text { (BLlrIge) Fenzl. }\end{array}$ & 92.73 & 6.36 & Tap & Sandy light Kastanozems \\
\hline $\begin{array}{l}\text { Halenia comiculata }(\mathrm{L} .) \\
\text { Comaz. }\end{array}$ & 5.00 & 2.00 & Tap & Meadow soil \\
\hline $\begin{array}{l}\text { Halerpestes ruthenica (Jacq.) } \\
\text { Ovez. }\end{array}$ & 6.62 & 6.92 & Fibrous & $\begin{array}{l}\text { Low wetland in steppe and desert } \\
\text { steppe }\end{array}$ \\
\hline $\begin{array}{l}\text { Haloxylon a mmodendron } \\
\text { (C.A.Mey.) Bunge. }\end{array}$ & 97.14 & 174.29 & Tap & Sand ( three years old) \\
\hline $\begin{array}{l}\text { Haloxylon a mmodendron (C. } \\
\text { A. Mey.) Bunge. }\end{array}$ & 170.00 & 46.25 & Tap & Dune (ten years old) \\
\hline $\begin{array}{l}\text { Haloxylon a mmodendron (C. } \\
\text { A. Mey.) Bunge }\end{array}$ & 563.64 & 181.82 & Tap & $\begin{array}{l}\text { Salinized fixed dune (six years } \\
\text { old) }\end{array}$ \\
\hline $\begin{array}{l}\text { Haloxylon persicum Bge. ex } \\
\text { Boiss. et Buhse. }\end{array}$ & 87.69 & 40.00 & Tap & Sandy desert in grassland \\
\hline $\begin{array}{l}\text { Haplophyllum dauricum (L.) } \\
\text { G. Don. }\end{array}$ & 12.57 & 4.86 & Tap & Kastanozems type of litho soils \\
\hline $\begin{array}{l}\text { Haplophyllum dauricum (L.) } \\
\text { G. Don. }\end{array}$ & 25.43 & 18.29 & Tap & Kastanozems \\
\hline $\begin{array}{c}\text { Hedysarum brachypterum } \\
\text { Bunge }\end{array}$ & 66.55 & 17.45 & Tap & $\begin{array}{l}\text { Typical steppe and desert steppe } \\
\text { sandy brown calcic soils }\end{array}$ \\
\hline Hedysarum frutcosum Pall. & 65.71 & 110.48 & Creeping & Fixed dune \\
\hline $\begin{array}{l}\text { Hedysarum fruticosum Pall. } \\
\text { Lignosum (Trautv.) Kitag. }\end{array}$ & 71.43 & 257.14 & Creeping & $\begin{array}{c}\text { Semi-mobile dune in Kubuqi } \\
\text { desert (three years old) }\end{array}$ \\
\hline $\begin{array}{l}\text { Hedysarum fruticosum Pall. } \\
\text { Lignosum (Trautv.) Kitag. }\end{array}$ & 102.63 & 121.58 & Creeping & Semi-fixed sand (six years old) \\
\hline $\begin{array}{l}\text { Hedysarum fruticosum Pall. } \\
\text { var. lignosum (Trautv.) Kitag. }\end{array}$ & 615.60 & 72.00 & Tap & Semi-mobiles sand \\
\hline $\begin{array}{c}\text { Hedysarum fruticosum Pall. } \\
\text { var. mongolicum Turcz. ex B. } \\
\text { Fedtsch. }\end{array}$ & 97.14 & 480.00 & Tap & Kubuqi desert \\
\hline Hedysarum gmelinii Ledeb. & 97.14 & 8.57 & Tap & Light Kastanozems \\
\hline Hedysarum gmelinii Ledeb. & 137.14 & 17.14 & Tap & Typical steppe Kastanozems \\
\hline $\begin{array}{l}\text { Hedysarum scoparium } \\
\text { Fisch.et Mey. }\end{array}$ & 87.10 & 197.42 & Tap & Kubuqi Desert \\
\hline $\begin{array}{l}\text { Hedysarum scoparium } \\
\text { Fisch.et Mey. }\end{array}$ & 164.71 & 200.00 & Tap & Tenggeli Desert ( three years old) \\
\hline $\begin{array}{c}\text { Helianthemum songaricum } \\
\text { Schrenk. }\end{array}$ & 8.88 & 11.25 & Tap & $\begin{array}{c}\text { Gravelly brown calcic soils in the } \\
\text { foot of a mountain }\end{array}$ \\
\hline $\begin{array}{l}\text { Helictotrichon schellianum } \\
\text { (Hack.) Kitag. }\end{array}$ & 37.00 & 10.00 & Fasciculate & $\begin{array}{l}\text { Mountain meadow steppe and } \\
\text { mountain steppe }\end{array}$ \\
\hline
\end{tabular}




\begin{tabular}{|c|c|c|c|c|}
\hline $\begin{array}{l}\text { Hemarthria altissima (Poir.) } \\
\text { Stapf et C. E. Hubb. }\end{array}$ & 11.50 & 5.25 & Rhizome & Sandy meadow soil \\
\hline Hemerocallis minor Mill & 18.00 & 12.43 & Fibrous & Dark Kastanozems \\
\hline $\begin{array}{l}\text { Herminium monorchis (L.) R. } \\
\text { Br. }\end{array}$ & 3.00 & 1.75 & Tuber & Meadow soil in low wetland \\
\hline $\begin{array}{l}\text { Herminium monorchis (L.) R. } \\
\text { Br. }\end{array}$ & 4.17 & 3.00 & Tuber & Meadow soil in reiverside \\
\hline $\begin{array}{c}\text { Heteropappus altaicus } \\
\text { (Willd.) Novopokr. }\end{array}$ & 8.10 & 8.20 & Tap & $\begin{array}{l}\text { Eutrie fluvisio in dry riverbed of } \\
\text { steppe desert }\end{array}$ \\
\hline $\begin{array}{l}\text { Heteropappus altaicus } \\
\text { (Willd.) Novopokr. }\end{array}$ & 8.64 & 2.91 & Tap & $\begin{array}{l}\text { Light Kastanozems in desert } \\
\text { steppe }\end{array}$ \\
\hline $\begin{array}{l}\text { Heteropappus altaicus } \\
\text { (Willd.) Novopokr. }\end{array}$ & 8.70 & 18.60 & Tap & Eroded slope by wind \\
\hline $\begin{array}{l}\text { Heteropappus meyendorffii } \\
\text { (Reg. et Maack) Komar. et } \\
\text { Klob. -Alis. }\end{array}$ & 12.00 & 17.33 & Tap & Aeolian soil in fixed sand \\
\hline Hierochloe glabra Trin. & 3.63 & 13.38 & Rhizome & Meadow Kastanozems \\
\hline $\begin{array}{l}\text { Hippolytia trifida (Turcz.) } \\
\text { Poljak. } \\
\end{array}$ & 18.00 & 13.40 & Tap & $\begin{array}{l}\text { Light brown calcic soils between } \\
\text { dunes }\end{array}$ \\
\hline $\begin{array}{l}\text { Hippolytia trifida (Turcz.) } \\
\text { Poljak. } \\
\end{array}$ & 31.82 & 21.36 & Tap & $\begin{array}{c}\text { Light Kastanozems in sandy high } \\
\text { plains }\end{array}$ \\
\hline $\begin{array}{l}\text { Hippolytia trifida (Turcz.) } \\
\text { Poljak. }\end{array}$ & 35.91 & 20.00 & Tap & Sandy light brown calcic soils \\
\hline $\begin{array}{l}\text { Hippolytia trifida (Turcz.) } \\
\text { Poljak. }\end{array}$ & 70.63 & 35.00 & Tap & $\begin{array}{l}\text { Light Kastanozems in desert } \\
\text { steppe }\end{array}$ \\
\hline $\begin{array}{l}\text { Hordeum brevisubulatum } \\
\text { (Trin.) Link. }\end{array}$ & 33.13 & 30.00 & Rhizome & Salinized meadow in valley \\
\hline Hvpecoum erectum L. & 40.63 & 19.38 & Tap & Fixed sand \\
\hline $\begin{array}{l}\text { Hylotelephium purpureum } \\
\text { (L.) Holub. }\end{array}$ & 9.27 & 7.91 & Tuber & Dark Kastanozems \\
\hline Incarvillea sinensis Lam. & 8.22 & 5.33 & Tap & Lowland of fixed sand \\
\hline Inula britanica $\mathrm{L}$. & 5.00 & 5.00 & Tap & Low wetland between dunes \\
\hline Inula britanica $\mathrm{L}$. & 11.55 & 8.36 & Tap & Flood plain \\
\hline $\begin{array}{c}\text { Inula britanica L.var. } \\
\text { Japonica (Thunb.) Franch. }\end{array}$ & 10.67 & 16.67 & Tap & Fluvo-aquic soils between dunes \\
\hline Inula lineariifolia Turcz. & 22.64 & 11.73 & Tap & Meadow \\
\hline $\begin{array}{l}\text { Inula salsoloides (Turcz.) } \\
\text { Ostenf. }\end{array}$ & 9.88 & 17.25 & Creeping & Aeolian soil \\
\hline $\begin{array}{l}\text { Inula salsoloides (Turcz.) } \\
\text { Ostenf. }\end{array}$ & 120.88 & 14.12 & Tap & Fixed sand \\
\hline Iris bungei Maxim. & 111.43 & 83.57 & Fasciculate & Light Kastanozems \\
\hline Iris dichotoma Pall. & 12.40 & 6.40 & Fibrous & $\begin{array}{c}\text { Sandy soil in riverside of } \\
\text { seasonal river }\end{array}$ \\
\hline Iris dichotoma Pall. & 18.25 & 7.00 & Fibrous & Dark Kastanozems \\
\hline $\begin{array}{l}\text { Iris lactea Pall. var. chinensis } \\
\text { (Fisch.) Koidz. }\end{array}$ & 80.00 & 35.56 & Fasciculate & Meadow soil \\
\hline Iris tenuifolia Pall. & 15.78 & 7.56 & Fasciculate & Desert steppe \\
\hline Iris tigridia Bunge. & 8.00 & 5.67 & Fasciculate & Upland meadow \\
\hline $\begin{array}{l}\text { Ixeridium chinense (Thunb.) } \\
\text { Tzvel. }\end{array}$ & 6.14 & 10.43 & Creeping & Humid sandy soil \\
\hline $\begin{array}{l}\text { Ixeridium graminifolium } \\
\text { (Ledeb.) Tzvel. }\end{array}$ & 45.00 & 4.55 & Tap & Fixed sand \\
\hline $\begin{array}{l}\text { Juncellus serotinus (Rottb.) C. } \\
\text { B. Clarke. }\end{array}$ & 20.00 & 28.75 & Rhizome & Bog soils \\
\hline Juncus bufonius L. & 4.38 & 3.25 & Rhizome & Bog soils in riverside \\
\hline Juncus effusus Linn. & 7.29 & 2.57 & Rhizome & Bog soils of lowland between \\
\hline
\end{tabular}




\begin{tabular}{|c|c|c|c|c|}
\hline & & & & dunes \\
\hline $\begin{array}{c}\text { Juncus gracilicaulis A. } \\
\text { Camus. }\end{array}$ & 32.50 & 18.75 & Rhizome & Swampy Meadow between dunes \\
\hline $\begin{array}{l}\text { Kalidium cuspidatum (Ung.- } \\
\text { Sternb.) Grub. }\end{array}$ & 86.25 & 53.75 & Tap & $\begin{array}{c}\text { Salinized Sandy soil between } \\
\text { dunes }\end{array}$ \\
\hline $\begin{array}{c}\text { Kalidium foliatum (Pall.) } \\
\text { Moq. }\end{array}$ & 17.25 & 9.25 & Tap & $\begin{array}{c}\text { Salinized meadow soil in steppe } \\
\text { and desert }\end{array}$ \\
\hline $\begin{array}{l}\text { Kalidium foliatum (Pall.) } \\
\text { Moq. }\end{array}$ & 100.00 & 71.11 & Tap & Saline soil of lowland \\
\hline Kalidium gracile Fenzl. & 17.40 & 18.00 & Tap & Sandy soil between dunes \\
\hline Kalidium gracile Fenzl. & 124.00 & 34.00 & Tap & Saline soil \\
\hline Kochia prostrata (L.) Schrad. & 147.50 & 20.00 & Tap & Meadow steppe \\
\hline $\begin{array}{l}\text { Kochia scoparia (L.) Schrad. } \\
\text { var. Sieversiana (Pall) } \\
\text { Ulbr.ex Aschers.et Graebn. }\end{array}$ & 42.75 & 15.00 & Tap & Kastanozems in base of hills \\
\hline $\begin{array}{c}\text { Kochia scoparia (L.) Schrad. } \\
\text { var. Sieversiana (pall.) } \\
\text { Ulbr.ex Aschers.et Graebn. }\end{array}$ & 54.38 & 10.00 & Tap & Salinized sand \\
\hline Koeleria cristata (L.) Pers. & 25.45 & 23.27 & Fasciculate & $\begin{array}{l}\text { Light Kastanozems in gentle } \\
\text { slope of hills }\end{array}$ \\
\hline $\begin{array}{l}\text { Kummerowia striata (Thunb.) } \\
\text { Schindl. }\end{array}$ & 6.92 & 4.46 & Tap & Fixed sand \\
\hline Lagochilus ilicifolius Bunge. & 21.43 & 25.00 & Tap & Sandy light Kastanozems \\
\hline $\begin{array}{l}\text { Lathyrus quinquenervius } \\
\text { (Miq.) Litv.et Kom. }\end{array}$ & 7.64 & 6.82 & Creeping & Meadow soil in riverside \\
\hline $\begin{array}{l}\text { Lathyrus quinquenervius } \\
\text { (Miq.) Litv.et Kom. }\end{array}$ & 16.20 & 38.40 & Creeping & $\begin{array}{c}\text { Meadow soil in terrace of } \\
\text { riverside }\end{array}$ \\
\hline Latuca tataricum (L.) D. C. & 25.56 & 32.22 & Creeping & $\begin{array}{l}\text { Fluvo-aquic soils in lowland } \\
\text { between dunes }\end{array}$ \\
\hline $\begin{array}{c}\text { Leontopodium } \\
\text { leontopodioides (Willd.) } \\
\text { Beauv. } \\
\end{array}$ & 7.27 & 7.27 & Fibrous & Meadow bog soils \\
\hline $\begin{array}{c}\text { Leontopodium } \\
\text { leontopodioides (Willd.) } \\
\text { Beauv. } \\
\end{array}$ & 10.33 & 7.00 & Fibrous & Sandy meadow soil \\
\hline Leonurus sibiricus L. & 28.50 & 38.50 & Tap & Sandy Kastanozems in hills \\
\hline Lepidium apetalum Willd. & 20.00 & 11.09 & Tap & Light Kastanozems in hills \\
\hline $\begin{array}{l}\text { Lepidium cartilagineum (J. } \\
\text { May.) Thell. }\end{array}$ & 11.20 & 8.60 & Creeping & Saline soil in steppe \\
\hline Lespedeza bicolor Turcz. & 102.56 & 35.90 & Tap & Light Kastanozems \\
\hline $\begin{array}{l}\text { Lespedeza davurica (Laxm.) } \\
\text { Schindl. }\end{array}$ & 67.64 & 28.36 & Tap & Slope in hills of typical steppe \\
\hline $\begin{array}{l}\text { Lespedeza juncea (linn. f.) } \\
\text { Pers. }\end{array}$ & 14.50 & 4.25 & Tap & $\begin{array}{c}\text { Dark Kastanozems in meadow } \\
\text { steppe }\end{array}$ \\
\hline Lespedeza potaninii Vass. & 7.86 & 4.29 & Tap & Aeolian sand \\
\hline Lespedeza potaninii Vass. & 116.84 & 56.84 & Tap & Semi-fixed sand \\
\hline $\begin{array}{l}\text { Leymus chinensis (Trin.) } \\
\text { Tzvel. }\end{array}$ & 72.50 & 41.67 & Rhizome & Meadow steppe \\
\hline $\begin{array}{l}\text { Leymus secalinus (Georgi) } \\
\text { Tzvel. } \\
\end{array}$ & 21.00 & 13.50 & Rhizome & Alluvial soils \\
\hline $\begin{array}{l}\text { Leymus secalinus (Georgi) } \\
\text { Tzvel. } \\
\end{array}$ & 26.11 & 18.33 & Rhizome & $\begin{array}{c}\text { Aeolian soil in dry riverbed } \\
\text { terraces }\end{array}$ \\
\hline $\begin{array}{l}\text { Leymus secalinus (Georgi) } \\
\text { Tzvel. }\end{array}$ & 43.33 & 37.33 & Rhizome & Loamy and sandy Kastanozems \\
\hline $\begin{array}{c}\text { Ligularia mongolica (Turcz.) } \\
\text { D C. }\end{array}$ & 21.82 & 20.00 & Fibrous & Dark Kastanozems \\
\hline
\end{tabular}




\begin{tabular}{|c|c|c|c|c|}
\hline Ligularia sibirica (L.) Cass. & 20.71 & 20.00 & Rhizome & Meadow soil \\
\hline Lilium pumilum D. C. & 20.20 & 8.00 & Tuber & $\begin{array}{l}\text { Gravelly dark Kastanozems in } \\
\text { slope }\end{array}$ \\
\hline Limonium aureum (L.) Hill. & 80.63 & 21.25 & Tap & Salinized meadow soil \\
\hline $\begin{array}{l}\text { Linaria vulgaris mill. subsp. } \\
\text { sinensis (Bebeaux) Hong. }\end{array}$ & 4.80 & 1.50 & Tap & Chernozems \\
\hline $\begin{array}{l}\text { Linaria vulgaris mill. subsp. } \\
\text { sinensis (Bebeaux) Hong. }\end{array}$ & 6.40 & 1.40 & Tap & Sandy Kastanozems in flood land \\
\hline Linum perenne L. & 9.91 & 2.00 & Tap & Stony chestnut soils in hills \\
\hline Lolium perenne L. & 12.22 & 15.56 & Fasciculate & Artificial grassland \\
\hline $\begin{array}{l}\text { Lomatogonium rotatum (L.) } \\
\text { Fries ex Num. }\end{array}$ & 2.91 & 1.64 & Tap & Meadow soil \\
\hline $\begin{array}{l}\text { Lysimachia baryrstachys } \\
\text { Brnge. }\end{array}$ & 33.13 & 22.50 & Tap & $\begin{array}{c}\text { Dark Kastanozems between } \\
\text { dunes }\end{array}$ \\
\hline Lysimachia thyrsiflora $\mathrm{L}$. & 20.00 & 29.50 & Rhizome & Bog soils \\
\hline Malva uertieillata L. & 42.22 & 12.22 & Tap & Skeletol soils \\
\hline Medicago falcata $\mathrm{L}$. & 36.50 & 48.00 & Tap & Aeolian soilin Drains \\
\hline Medicago falcata $\mathrm{L}$. & 69.17 & 66.67 & Tap & Riverside \\
\hline Medicago lupulina L. & 2.00 & 2.00 & Tap & Meadow soil in riverside \\
\hline Medicago lupulina L. & 9.00 & 2.30 & Tap & Dark Kastanozems in hill \\
\hline Medicago sativa $\mathrm{L}$. & 467.00 & 35.67 & Tap & Bog soils in meadow \\
\hline Medicago varia Martyn. & 7.50 & 4.88 & Tap & $\begin{array}{c}\text { Dark Kastanozems in meadow } \\
\text { steppe }\end{array}$ \\
\hline $\begin{array}{l}\text { Melilotoides ruthenica (L.) } \\
\text { Sojak. }\end{array}$ & 30.00 & 19.88 & Tap & Aeolian sand in hills \\
\hline $\begin{array}{l}\text { Melilotoides ruthenica ( L.) } \\
\text { Sojak. } \\
\end{array}$ & 47.00 & 13.50 & Tap & Light Kastanozems \\
\hline $\begin{array}{l}\text { Melilotoides ruthenica (L.) } \\
\text { Sojak. }\end{array}$ & 83.03 & 9.66 & Tap & Light Kastanozems \\
\hline $\begin{array}{l}\text { Melilotoides ruthenica (L.) } \\
\text { Sojak. }\end{array}$ & 96.00 & 23.00 & Tap & Kastanozems in typical steppe \\
\hline $\begin{array}{l}\text { Melilotoides ruthenica }(\mathrm{L} .) \\
\text { Sojak. }\end{array}$ & 105.00 & 24.75 & Tap & Sandy Kastanozems \\
\hline $\begin{array}{l}\text { Melilotus dentata (Waldst. et } \\
\text { Kit.) Pers. }\end{array}$ & 12.00 & 15.43 & Tap & Meadow soil in steppe \\
\hline $\begin{array}{l}\text { Melilotus dentata (Waldst. et } \\
\text { Kit.) Pers. }\end{array}$ & 36.67 & 50.00 & Tap & Floodland in meadow soil \\
\hline $\begin{array}{l}\text { Melilotus officinalis (Linn.) } \\
\text { Pall. }\end{array}$ & 51.11 & 48.89 & Tap & $\begin{array}{l}\text { Fluvo-aquic soils in lowland } \\
\text { between dunes of meadow }\end{array}$ \\
\hline Mentha haplocalyx Briq. & 1.79 & 1.47 & Tap & Sandy Kastanozems \\
\hline Mentha haplocalyx Briq. & 16.86 & 9.71 & Tap & Low wetland near river \\
\hline $\begin{array}{l}\text { Messerschmidia sibirica L. } \\
\text { var. angustior (D. C.) W. T. } \\
\text { Wang. }\end{array}$ & 75.00 & 18.89 & Creeping & Meadow soil in low wetland \\
\hline Myricaria platyphylla Maxim & 202.78 & 330.56 & Tap & $\begin{array}{l}\text { Mobile dune in desert steppe and } \\
\text { steppe desert }\end{array}$ \\
\hline $\begin{array}{l}\text { Neopallasia pectinata (Pall.) } \\
\text { Poljak. }\end{array}$ & 5.68 & 3.64 & Tap & Litho soils in hills \\
\hline $\begin{array}{l}\text { Neopallasia pectinata (Pall.) } \\
\text { Poljak. }\end{array}$ & 10.93 & 1.71 & Tap & Light Kastanozems in slope \\
\hline Nitraria tangutorum Bobr. & 186.43 & 171.43 & Tap & Aeolian soils \\
\hline Nitrraria sibirica Pall. & 37.94 & 79.41 & Tap & Aeolian soils in sub-fixed sand \\
\hline $\begin{array}{c}\text { Olgaea leucophylla (Turcz.) } \\
\text { Iljin. }\end{array}$ & 40.00 & 12.67 & Tap & Fixed sand \\
\hline $\begin{array}{l}\text { Olgaea lomonossowii } \\
\text { (Trautv.) Iljin. }\end{array}$ & 68.62 & 2.19 & Tap & $\begin{array}{l}\text { Brown calcic soils in overlying } \\
\text { sand }\end{array}$ \\
\hline
\end{tabular}




\begin{tabular}{|c|c|c|c|c|}
\hline $\begin{array}{l}\text { Orobanche coerulescens } \\
\text { Steph. }\end{array}$ & 37.50 & 24.75 & Fibrous & Dune in steppe \\
\hline $\begin{array}{c}\text { Orobanche pycnostachya } \\
\text { Hance }\end{array}$ & 7.20 & 3.00 & Fibrous & Dune in steppe \\
\hline $\begin{array}{c}\text { Orostachys fimbriatu } \\
\text { s(Turcz.) Berger }\end{array}$ & 4.11 & 4.67 & Tap & Dark Kastanozems in hills \\
\hline $\begin{array}{l}\text { Orostachys malacophyllus } \\
\text { (Pall.) Fisch. }\end{array}$ & 8.22 & 9.56 & Tap & $\begin{array}{c}\text { Dark Kastanozems between } \\
\text { dunes }\end{array}$ \\
\hline Oxytropis aciphylla Ledeb. & 105.75 & 16.50 & Tap & $\begin{array}{l}\text { Gravelly Kastanozems in desert } \\
\text { steppe }\end{array}$ \\
\hline Oxytropis bicolor Bunge & 20.00 & 12.67 & Tap & Gravelly sand in arid high plains \\
\hline Oxytropis bicolor Bunge & 126.36 & 14.55 & Tap & Sandy steppe sand \\
\hline Oxytropis filiformis D. C. & 78.75 & 15.00 & Tap & $\begin{array}{l}\text { Gravelly sand in hills of forest or } \\
\text { typical steppe }\end{array}$ \\
\hline Oxytropis glabra (Lam.) D. C. & 68.18 & 34.09 & Tap & Low wetland in desert steppe \\
\hline $\begin{array}{c}\text { Oxytropis grandiflora (Pall.) } \\
\text { D. C. }\end{array}$ & 51.88 & 43.13 & Tap & Meadow steppe in hills \\
\hline $\begin{array}{c}\text { Oxytropis leptophylla (Pall.) } \\
\text { D. C. }\end{array}$ & 62.73 & 17.73 & Tap & $\begin{array}{l}\text { Gravelly sand in hills of forest or } \\
\text { typical steppe }\end{array}$ \\
\hline $\begin{array}{c}\text { Oxytropis myriophylla (Pall.) } \\
\text { D. C. }\end{array}$ & 36.88 & 9.38 & Tap & Sandy \\
\hline $\begin{array}{c}\text { Oxytropis myriophylla (Pall.) } \\
\text { D. C. }\end{array}$ & 133.85 & 51.92 & Tap & $\begin{array}{c}\text { Dark Kastanozems in meadow } \\
\text { steppe }\end{array}$ \\
\hline Oxytropis racemosa Turcz. & 140.00 & 39.12 & Tap & Semi-fixed sand \\
\hline Oxytropis racemosa Turcz. & 160.00 & 26.67 & Tap & Mobile dune \\
\hline Oxytropis squammulosa D. C. & 165.52 & 55.86 & Tap & $\begin{array}{l}\text { Brown calcic soils in desert } \\
\text { steppe }\end{array}$ \\
\hline Oxytropis subfalcata Hance & 19.33 & 8.33 & Tap & Sandy Kastanozems in hgh plains \\
\hline Paeonia lachfiona Pall. & 30.86 & 11.14 & Tap & Aeolian sandy soils \\
\hline Papaver nudicaule L. & 51.60 & 10.20 & Tap & Low wetland in riverside \\
\hline Parnassia palustris L. & 2.00 & 2.00 & Fibrous & Bog soils in riverside \\
\hline $\begin{array}{c}\text { Patrinia rupestris (Pall.). } \\
\text { Juss. Subsp. scabra (Bunge) } \\
\text { H. J. Wang }\end{array}$ & 18.33 & 4.67 & Tap & Aeolian sandy soils \\
\hline Pcnzeria alaschanica Kupr. & 20.25 & 2.81 & Tap & $\begin{array}{l}\text { Sandy brown calcic soils in dry } \\
\text { riverbed }\end{array}$ \\
\hline Pcnzeria alaschanica Kupr. & 96.00 & 33.00 & Tap & Fixed sand \\
\hline Pedicularis striata Pall. & 31.50 & 22.00 & Tap & Dark Kastanozems in hills \\
\hline $\begin{array}{l}\text { Pedicularis venusta Schangan } \\
\text { ex Bunge }\end{array}$ & 15.00 & 15.71 & Creeping & Dark meadow soil in riverside \\
\hline Pedicularis verticillata L. & 5.63 & 2.13 & Tap & Meadow soil in riverside \\
\hline $\begin{array}{l}\text { Peganum multisectum } \\
\text { (Maxim.) Bobr. }\end{array}$ & 60.00 & 131.05 & Creeping & Semi-fixed sand \\
\hline Peganum nigellastrum Bunge & 157.14 & 38.57 & Creeping & Sandy brown calcic soils \\
\hline $\begin{array}{l}\text { Pennisetum centrasiaticum } \\
\text { Tzvel. }\end{array}$ & 21.38 & 15.38 & Rhizome & Sandy meadow in fixed sand \\
\hline Phalaris arundinacea L. & 8.33 & 7.67 & Rhizome & Swampy meadow in hills \\
\hline Phlomis tuberosa L. & 26.80 & 4.40 & Tuber & Skeletol soils in hills \\
\hline $\begin{array}{l}\text { Phragmites australis (Cav.) } \\
\text { Trin. ex Steud. }\end{array}$ & 23.00 & 32.00 & Rhizome & Wetlands \\
\hline Plantago asiatica $\mathrm{L}$. & 19.64 & 20.00 & Fibrous & Meadow soil \\
\hline Plantago depressa Willd. & 7.40 & 1.80 & Tap & Meadow soil \\
\hline $\begin{array}{l}\text { Plantago lessingii Fisch. et } \\
\text { Mey. }\end{array}$ & 7.38 & 7.50 & Tap & Brown calcic soils in hills \\
\hline $\begin{array}{c}\text { Plantago lessingii Fisch. et } \\
\text { Mey }\end{array}$ & 25.50 & 2.25 & Tap & Light Kastanozems \\
\hline
\end{tabular}




\begin{tabular}{|c|c|c|c|c|}
\hline Plantago minuta Pall. & 48.13 & 12.50 & Tap & $\begin{array}{l}\text { Halophytic meadow in desert } \\
\text { steppe }\end{array}$ \\
\hline $\begin{array}{l}\text { Platycodon grandiflorus } \\
\text { (Jacq.) A. D C. }\end{array}$ & 34.44 & 21.11 & Tap & $\begin{array}{c}\text { Meadow dark Kastanozems } \\
\text { between dunes }\end{array}$ \\
\hline $\begin{array}{c}\text { Poa botryoides (Griseb.) } \\
\text { Roshev }\end{array}$ & 39.17 & 18.33 & Fasciculate & Lowland in Between dunes \\
\hline Poa nemoralis L. & 5.75 & 8.25 & Rhizome & Woodland \\
\hline Poa pratensis L. & 9.75 & 12.25 & Rhizome & Meadow steppe \\
\hline $\begin{array}{c}\text { Poa sphondylodes Trin,ex } \\
\text { Bunge }\end{array}$ & 23.40 & 16.20 & Fasciculate & $\begin{array}{c}\text { Dark Kastanozems in meadow } \\
\text { steppe }\end{array}$ \\
\hline $\begin{array}{c}\text { Poa sphondylodes Trin,ex } \\
\text { Bunge }\end{array}$ & 44.80 & 30.40 & Fasciculate & Kastanozems \\
\hline Poa subfastigiata Trin. & 10.80 & 13.20 & Rhizome & Meadow in valley \\
\hline Polygala tenuifolfa I, Willd. & 7.18 & 1.09 & Tap & Aeolian soils \\
\hline Polygala tenuifolfa I, Willd. & 11.50 & 1.80 & Tap & Desert \\
\hline Polygala tenuifolfa I, Willd. & 18.00 & 10.00 & Tap & Skeletol soils in hills \\
\hline $\begin{array}{c}\text { Polygonatum sibiricum } \\
\text { Delar.ex Recdoute }\end{array}$ & 30.00 & 43.13 & Rhizome & Dark Kastanozems \\
\hline $\begin{array}{c}\text { Polygonum alopecuroides } \\
\text { Tuecz. ex Besser } \\
\end{array}$ & 10.00 & 7.67 & Fibrous & Meadow \\
\hline Polygonum aviculare L. & 9.54 & 2.31 & Tap & $\begin{array}{c}\text { Meadow soil in low wetland of } \\
\text { riverside }\end{array}$ \\
\hline Polygonum divaricatum $\mathrm{L}$. & 20.50 & 5.50 & Tap & Aeolian sand \\
\hline Polygonum sibiricum Laxm. & 32.25 & 48.75 & Rhizome & $\begin{array}{c}\text { Meadow soil in riverside } \\
\text { Salinized } \\
\end{array}$ \\
\hline Portulace oleracea L. & 6.70 & 1.20 & Tap & Kastanozems \\
\hline $\begin{array}{c}\text { Potamogeton panormitanus } \\
\text { Biv. }\end{array}$ & 14.25 & 5.25 & Rhizome & Swampy land \\
\hline Potaninia mongolica Marim. & 42.27 & 128.18 & Tap & Grey brown desert soil \\
\hline $\begin{array}{l}\text { Potenilla vertcillaris ex Willd } \\
\text {. }\end{array}$ & 8.60 & 15.60 & Tap & Sandy black soils in slope \\
\hline $\begin{array}{c}\text { Potenilla vertcillaris ex Willd } \\
\text {. }\end{array}$ & 18.30 & 21.30 & Tap & Kastanozems litho soils \\
\hline Potentilla betonicifolia Poir. & 21.25 & 19.25 & Tap & Litho soils in hills \\
\hline Potentilla acaulis L. & 11.56 & 6.44 & Creeping & Chernozems \\
\hline Potentilla acaulis $\mathrm{L}$. & 24.44 & 12.00 & Creeping & $\begin{array}{l}\text { Brown earth type of } \\
\text { Kastanozems }\end{array}$ \\
\hline Potentilla anserina L. & 15.64 & 7.82 & Tuber & Low wetland in riverside \\
\hline Potentilla anserina $\mathrm{L}$. & 19.88 & 15.00 & Tuber & Bog soils in meadow \\
\hline Potentilla anserina $\mathrm{L}$. & 22.00 & 26.00 & Tuber & Aeolian sand indry riverbed \\
\hline Potentilla bifurca L. & 21.75 & 6.00 & Creeping & $\begin{array}{l}\text { Loamy and sandy typical } \\
\text { Kastanozems in vally }\end{array}$ \\
\hline $\begin{array}{l}\text { Potentilla bifurca L. var. } \\
\text { major Ledeb. }\end{array}$ & 164.29 & 87.14 & Creeping & Light Kastanozems \\
\hline $\begin{array}{l}\text { Potentilla flagellaris Willd.ex } \\
\text { Schlecht }\end{array}$ & 10.64 & 6.27 & Fibrous & $\begin{array}{l}\text { Brown soil between dunes in } \\
\text { hills }\end{array}$ \\
\hline $\begin{array}{l}\text { Potentilla parvifolia Fisch.ap. } \\
\text { Lehm. }\end{array}$ & 19.33 & 15.11 & Tap & Litho soils in hills \\
\hline $\begin{array}{c}\text { Potentilla tanacetifolia Willd. } \\
\text { ex Schlecht }\end{array}$ & 32.67 & 13.00 & Tap & Light Kastanozems in slope \\
\hline $\begin{array}{l}\text { Potentilla tanacetifolia Willd. } \\
\text { ex Schlecht }\end{array}$ & 40.80 & 6.40 & Tap & Light saline soil in low wetland \\
\hline $\begin{array}{c}\text { Potentilla strigosa Pall. ex } \\
\text { Pursh } \\
\end{array}$ & 15.40 & 17.60 & Tap & Litho soils in hills \\
\hline $\begin{array}{c}\text { Psammochloa villosa (Trin.) } \\
\text { Bor }\end{array}$ & 120.00 & 36.36 & Rhizome & Mobile dune \\
\hline
\end{tabular}




\begin{tabular}{|c|c|c|c|c|}
\hline $\begin{array}{c}\text { Ptilotrichum conescens (DC.) } \\
\text { C. A. Mev. }\end{array}$ & 36.86 & 2.57 & Tap & Saline soil in meadow \\
\hline $\begin{array}{l}\text { Puccinellia distans (Jacq.) } \\
\text { Parl. }\end{array}$ & 3.27 & 2.45 & Fasciculate & $\begin{array}{l}\text { Salinized meadow or water area } \\
\text { in riverside }\end{array}$ \\
\hline $\begin{array}{c}\text { Puccinellia hauptiana (Trin.) } \\
\text { Krecz }\end{array}$ & 11.08 & 14.46 & Rhizome & $\begin{array}{l}\text { Swampy meadow in riverside } \\
\text { sand }\end{array}$ \\
\hline $\begin{array}{l}\text { Pucnellia tenuiflora (Turcz.) } \\
\text { Scribn. et Merr. }\end{array}$ & 14.40 & 6.60 & Fasciculate & $\begin{array}{l}\text { Fluvo-aquic soils in low wetland } \\
\text { saline }\end{array}$ \\
\hline $\begin{array}{l}\text { Pucnellia tenuiflora (Turcz.) } \\
\text { Scribn. et Merr. }\end{array}$ & 31.36 & 21.82 & Fasciculate & $\begin{array}{l}\text { Fluvo-aquic soils in lowland } \\
\text { between dunes }\end{array}$ \\
\hline $\begin{array}{c}\text { Pucnellia tenuiflora (Turcz.) } \\
\text { Scribn.et Merr. }\end{array}$ & 36.67 & 23.89 & Fasciculate & Salinized sandy \\
\hline $\begin{array}{l}\text { Pugionium cornutum (L.) } \\
\text { Gaertn. }\end{array}$ & 127.78 & 36.67 & Tap & Dune \\
\hline $\begin{array}{c}\text { Pulsatilla turczanninovii } \\
\text { Kryl.et Serg. }\end{array}$ & 12.86 & 12.57 & Tap & $\begin{array}{c}\text { Typical light Kastanozems in } \\
\text { hills }\end{array}$ \\
\hline $\begin{array}{l}\text { Pycreus globosus (AlI.) } \\
\text { Reichb. }\end{array}$ & 5.65 & 3.53 & Rhizome & Meadow soil between dunes \\
\hline $\begin{array}{c}\text { Reaumuria songarica (Pall.) } \\
\text { Maxim. }\end{array}$ & 14.13 & 6.00 & Tap & Brown calcic soils \\
\hline Rheum undulatum L. & 15.78 & 4.89 & Tap & Stony slope in hills \\
\hline Rheum uninerve Maxim. & 17.25 & 8.25 & Tuber & $\begin{array}{l}\text { Sandy and gray desert soils in } \\
\text { high plains }\end{array}$ \\
\hline $\begin{array}{c}\text { Rorippa islandica ( Oed.) } \\
\text { Borb }\end{array}$ & 51.50 & 7.50 & Tap & Fluvo-quic soil in low wetland \\
\hline Rubia cordifolia $\mathrm{L}$. & 61.00 & 22.00 & Tap & $\begin{array}{l}\text { Between dunes Low wetland } \\
\text { meadow soil }\end{array}$ \\
\hline Rumex acetosella L. & 190.00 & 32.00 & Tap & Sand in meadow steppe \\
\hline Sagittaria trifolia L. & 9.86 & 16.29 & Tuber & Bog soils in riverside \\
\hline Salicornia europaea L. & 9.00 & 4.00 & Tap & $\begin{array}{l}\text { Lowland of Fixed dune between } \\
\text { dunes }\end{array}$ \\
\hline $\begin{array}{c}\text { Salix gordejevii Y. L.Chang et } \\
\text { Skv. }\end{array}$ & 142.86 & 985.71 & Tap & Semi-fixed dune in steppe \\
\hline $\begin{array}{c}\text { Salix gordejevii Y. L.Chang et } \\
\text { Skv. }\end{array}$ & 350.0 & 2000.0 & Tap & Semi-fixed dune in steppe \\
\hline $\begin{array}{c}\text { Salix psammophila C. Wang } \\
\text { et Ch. Y. Yang }\end{array}$ & 24.83 & 7.00 & Tap & Fixed sand \\
\hline Salsola collina Pall. & 19.43 & 10.00 & Tap & Light Kastanozems in hills \\
\hline $\begin{array}{c}\text { Salsola laricifolia Turcz.ex } \\
\text { Litv. }\end{array}$ & 83.75 & 76.25 & Tap & Light brown calcic soils in hills \\
\hline Salsola passerina Bunge & 29.11 & 14.89 & Tap & Litho soils in hills \\
\hline Salsola passerina Bunge & 37.50 & 17.50 & Tap & $\begin{array}{l}\text { Brown calcic soils in ground } \\
\text { between dunes }\end{array}$ \\
\hline Salsola passerina Bunge & 80.00 & 80.00 & Tap & Saline soil \\
\hline Sanguisorba offiacinalis L. & 13.25 & 11.25 & Tap & Meadow soil in riverside \\
\hline $\begin{array}{l}\text { Saposhnikovia divaricata } \\
\text { (Turcz.) Schischk. }\end{array}$ & 130.00 & 24.00 & Tap & $\begin{array}{l}\text { Typical Kastanozems in loamy } \\
\text { and sandy ground }\end{array}$ \\
\hline $\begin{array}{c}\text { Sarcozygium xanthoxylon } \\
\text { Bunge }\end{array}$ & 37.86 & 41.43 & Tap & Desert soil \\
\hline Saussurea amara (L.) D. C. & 65.00 & 63.75 & Tap & Light Kastanozems \\
\hline Saussurea amara (L.) D. C. & 105.00 & 16.20 & Tap & Typical Kastanozems \\
\hline $\begin{array}{c}\text { Saussurea japonica (Thunb.) } \\
\text { D. C. }\end{array}$ & 40.67 & 16.67 & Tap & Light Kastanozems \\
\hline $\begin{array}{l}\text { Saussurea salsa (Pall.) } \\
\text { Spreng. } \\
\end{array}$ & 10.36 & 7.27 & Tap & Meadow soil in Salinized sand \\
\hline Scabiosa comosa fisch. ex & 20.63 & 8.25 & Tap & Fixeds and \\
\hline
\end{tabular}




\begin{tabular}{|c|c|c|c|c|}
\hline Roem.et Schult. & & & & \\
\hline $\begin{array}{c}\text { Scabiosa comosa fisch. ex } \\
\text { Roem.et Schult. }\end{array}$ & 24.89 & 4.44 & Tap & Aeolian soils \\
\hline $\begin{array}{l}\text { Scabiosa comosa fisch. ex } \\
\text { Roem.et Schult. }\end{array}$ & 29.57 & 19.71 & Tap & Sand at the base of fixed dune \\
\hline $\begin{array}{c}\text { Scirpus planiculmis Fr. } \\
\text { Schmidt }\end{array}$ & 9.00 & 17.50 & Tuber & Bog soils in riverside \\
\hline $\begin{array}{l}\text { Scirpus tabernaemontani } \\
\text { Gmel. }\end{array}$ & 84.55 & 70.91 & Rhizome & Bog soils \\
\hline Scorzonera albicaulis Bunge & 60.00 & 38.75 & Tap & Dark Kastanozems \\
\hline Scorzonera muriculata Chang & 17.00 & 14.00 & Creeping & $\begin{array}{c}\text { Light brown calcic soils in } \\
\text { overlying sand }\end{array}$ \\
\hline Scorzonera muriculata Chang & 18.80 & 2.20 & Creeping & Kastanozems \\
\hline Scutellaria baicalensis Georgi & 15.14 & 18.86 & Creeping & Typical Kastanozems in hills \\
\hline $\begin{array}{c}\text { Scutellaria scordifolia Fisch. } \\
\text { ex Schrank }\end{array}$ & 2.86 & 4.43 & Creeping & Kastanozems \\
\hline Scutellaria viscidula Bunge & 14.00 & 19.50 & Creeping & Kastanozems between duned \\
\hline Sedum aizoon $\mathrm{L}$. & 14.89 & 6.22 & Tap & Podzolic soils in hills \\
\hline $\begin{array}{l}\text { Sedum aizoon L.f. } \\
\text { angusifolium Franch. }\end{array}$ & 8.63 & 2.50 & Tap & Kastanozems in high plains \\
\hline Senecio argunensis Turcz. & 21.67 & 15.00 & Fibrous & Fixed dune \\
\hline Serratula polycephala Iljin & 98.00 & 32.00 & Tap & Steppe \\
\hline Setaria glauca (L.) Beauv. & 14.86 & 11.43 & Fasciculate & Dark Kastanozems \\
\hline Setaria viridis (L.) Beauv. & 11.43 & 15.71 & Fasciculate & Dark Kastanozems \\
\hline Setaria viridis (L.) Beauv. & 30.56 & 23.33 & Fasciculate & Fixed sand \\
\hline Silene jenisseensis Willd. & 36.43 & 20.57 & Tap & Skeletol soils in hills \\
\hline Silene repens Patr. & 113.33 & 33.33 & Tap & Base of mobile dune \\
\hline Solanum septemlobum Bunge & 120.00 & 66.00 & Tap & Sub-fixed sand \\
\hline Sonchus arvensis L. & 37.08 & 13.33 & Creeping & Salinized and sandy lowland \\
\hline Sophora alopecuroides L. & 21.86 & 33.86 & Creeping & Desert and steppe desert \\
\hline Sophora flavescens Soland & 42.00 & 4.00 & Tap & Steppe or Sandy steppe \\
\hline Sparganium simplex Huds. & 28.75 & 22.50 & Rhizome & Bog soils \\
\hline $\begin{array}{l}\text { Sparganium stoloniferum } \\
\text { (Graebn.) Buch. }\end{array}$ & 3.13 & 9.38 & Tuber & Bog soils \\
\hline $\begin{array}{c}\text { Sphaerophysa salsula (Pall.) } \\
\text { D. C. }\end{array}$ & 20.75 & 25.00 & Creeping & Meadow soil in low wetland \\
\hline Spiraea pubescens Turcz. & 10.67 & 11.83 & Tap & Podzolic soils \\
\hline $\begin{array}{c}\text { Spiranthes sinensis (Pers.) } \\
\text { Ames }\end{array}$ & 7.17 & 3.33 & Tuber & Wetland in forest \\
\hline $\begin{array}{l}\text { Stellaria dichotoma } \mathrm{L} . \text { var. } \\
\text { lanceolata Bunge }\end{array}$ & 14.50 & 7.25 & Tap & Semi-fixed dune \\
\hline $\begin{array}{l}\text { Stellaria dichotoma L. var } \\
\text { lanceolata Bunge }\end{array}$ & 77.00 & 10.50 & Tap & Sand in windward slope \\
\hline Stellaria discolor Turcz. & 5.80 & 1.80 & Tap & Kastanozems in hills \\
\hline Stellaria discolor Turcz. & 7.00 & 2.60 & Tap & Chernozems \\
\hline Stellera chomaejasme L. & 68.18 & 16.82 & Tap & Salinized Kastanozems \\
\hline Stellera chomaejasme L. & 93.08 & 27.69 & Tap & Dark Kastanozems \\
\hline $\begin{array}{l}\text { Stemmacantha uniflora (L.) } \\
\text { D. C. Dittrich }\end{array}$ & 34.91 & 16.09 & Tap & Litho soils in hills \\
\hline Stipa baicalensis Roshev. & 113.44 & 55.31 & Fasciculate & Dark Kastanozems \\
\hline Stipa breviflora Griseb. & 4.80 & 6.50 & Fasciculate & Desert steppe \\
\hline Stipa bungeana Trin. & 14.29 & 9.39 & Fasciculate & Typical steppe \\
\hline Stipa glareosa P. Smirn. & 50.00 & 27.78 & Fasciculate & $\begin{array}{l}\text { Gritty brown calcic soils in high } \\
\text { plains }\end{array}$ \\
\hline Stipa grandis P. Smirn. & 11.25 & 8.88 & Fasciculate & Kastanozems \\
\hline
\end{tabular}




\begin{tabular}{|c|c|c|c|c|}
\hline Stipa grandis P. Smirn. & 102.67 & 70.67 & Fasciculate & Dark Kastanozems \\
\hline Stipa purpurea Griseb. & 88.50 & 48.00 & Fasciculate & Meadow soils in alpine steppe \\
\hline $\begin{array}{l}\text { Stipa sareptana } \text { Becker var. } \\
\text { krylovii (Roshev.) P. C. Kuo } \\
\text { et Y. H. Sun }\end{array}$ & 42.18 & 16.00 & Fasciculate & Typical steppe \\
\hline $\begin{array}{l}\text { Stipa tianschanica Roshev. } \\
\text { Var. gobica (Roshev.) P. C. } \\
\text { Kuo et Y. H. Sun }\end{array}$ & 101.11 & 42.22 & Fasciculate & Sandy brown calcic soils \\
\hline $\begin{array}{l}\text { Stipa tianschanica } \\
\text { Roshev.var. klemenzii } \\
\text { (Roshev.) Norl. }\end{array}$ & 30.00 & 12.55 & Fasciculate & Sandy Kastanozems \\
\hline $\begin{array}{l}\text { Stipa tianschanica } \\
\text { Roshev.var. klemenzii } \\
\text { (Roshev.) Norl. }\end{array}$ & 87.10 & 61.94 & Fasciculate & $\begin{array}{l}\text { Light Kastanozems in desert } \\
\text { steppe and typical steppe }\end{array}$ \\
\hline $\begin{array}{l}\text { Suaeda glauca (Bunge) } \\
\text { Bunge }\end{array}$ & 12.11 & 8.33 & Tap & Desert soil \\
\hline Tamarix ramoslssima Ledeb. & 102.78 & 161.11 & Tap & Salinized soil in desert \\
\hline Tamarlx chinensis Lour. & 92.00 & 50.00 & Tap & Sub-fixeds \\
\hline Taraxacum asiaticum Dahlst. & 98.92 & 11.35 & Tap & Base of dune \\
\hline $\begin{array}{c}\text { Taraxacum borealisinense } \\
\text { Kitam. }\end{array}$ & 26.09 & 5.22 & Tap & Sandy meadow soil \\
\hline $\begin{array}{c}\text { Taraxacum borealisinense } \\
\text { Kitam. }\end{array}$ & 67.17 & 11.09 & Tap & Salinized meadow soil \\
\hline Taraxacum ohwianum Kitam. & 7.44 & 3.60 & Tap & Low wetland \\
\hline Thalictrum petaloideum $\mathrm{L}$. & 14.67 & 48.67 & Rhizome & $\begin{array}{c}\text { Light Kastanozems in desert } \\
\text { steppe }\end{array}$ \\
\hline Thalictrum petaloideum $\mathrm{L}$. & 15.50 & 13.75 & Rhizome & Aeolian sandy soils \\
\hline $\begin{array}{l}\text { Thalictrum petaloideum } \mathrm{L} . \\
\text { var. supradecompositum } \\
\text { (Nakai) Kitag. }\end{array}$ & 21.43 & 19.29 & Fibrous & Dark Kastanozems in hills \\
\hline Thalictrum simplex $\mathrm{L}$. & 4.38 & 5.75 & Fibrous & Dark Kastanozems \\
\hline $\begin{array}{c}\text { Thalictrum squarrosum } \\
\text { staph.ex Willd. } \\
\end{array}$ & 15.14 & 16.00 & Rhizome & Meadow soil \\
\hline $\begin{array}{l}\text { Thalictrum squarrosum } \\
\text { staph.ex Willd }\end{array}$ & 78.32 & 99.79 & Rhizome & Light Kastanozems \\
\hline Thermopsis lanceolata $\mathrm{R} . \mathrm{Br}$. & 146.67 & 37.78 & Creeping & Dark Kastanozems \\
\hline Thermopsis lanceolata $\mathrm{R} . \mathrm{Br}$. & 248.89 & 62.22 & Creeping & Semi-fixed sand \\
\hline Thymus mongollcus Ronn. & 11.14 & 6.71 & Tap & $\begin{array}{c}\text { Dark Kastanozems between } \\
\text { dunes }\end{array}$ \\
\hline Thymus mongollcus Ronn. & 12.33 & 19.00 & Tap & Semi-fixed sand \\
\hline Tragus racemosus (L.) AIl. & 14.00 & 13.50 & Fasciculate & $\begin{array}{c}\text { Sandy Kastanozems in slope of } \\
\text { hills }\end{array}$ \\
\hline Tribulus terrestris L. & 21.40 & 9.60 & Tap & Kastanozems in hills \\
\hline Trifolium lupinaster L. & 8.67 & 3.33 & Tap & $\begin{array}{l}\text { Swampy meadow soil in meadow } \\
\text { steppe }\end{array}$ \\
\hline Triglochin palustre $\mathrm{L}$. & 8.73 & 9.55 & Rhizome & Meadow-riverside bog soils \\
\hline Tugarinovia mongolica Iljin & 59.21 & 17.37 & Tap & Stony light Kastanozems \\
\hline Urtica cannabina $\mathrm{L}$. & 36.00 & 15.00 & Tap & $\begin{array}{c}\text { Stony and gravelly slope in forest } \\
\text { steppe }\end{array}$ \\
\hline Veratrum nigrum L. & 7.40 & 3.80 & Tuber & Under the shrub in meadow \\
\hline Veratrum nigrum $\mathrm{L}$. & 30.67 & 13.78 & Tuber & Low wetland in meadow \\
\hline Veronica dahurica Stev. & 8.50 & 6.83 & Fibrous & Chernozems in hills \\
\hline Veronica incana $\mathrm{L}$. & 6.56 & 3.44 & Fibrous & Meadow chernozems in vally \\
\hline $\begin{array}{l}\text { Veronica linariifolia Pall. ex } \\
\text { Link }\end{array}$ & 13.00 & 16.00 & Fibrous & Skeletol soils \\
\hline Vicia amoena Fisch. ex D. C. & 17.78 & 10.44 & Creeping & Chernozems in meadow steppe \\
\hline
\end{tabular}




\begin{tabular}{|c|c|c|c|c|}
\hline $\begin{array}{c}\text { Vicia amoena Fisch. var. } \\
\text { Oblongifolia } \text { Regel. }\end{array}$ & 47.78 & 23.33 & Creeping & Meadow steppe and meadow \\
\hline $\begin{array}{c}\text { Viola yedoensis Makino } \\
\begin{array}{c}\text { Xanthium sibiricum Patrin ex } \\
\text { Widder }\end{array}\end{array}$ & 14.50 & 2.00 & Tap & Kastanozems in alluvial plain \\
\hline $\begin{array}{c}\text { Youngia tenuicaulis } \text { (Babcock } \\
\text { et Stebbins) Czer. }\end{array}$ & 19.64 & 7.82 & Tap & Meadow soil \\
\hline
\end{tabular}

